\title{
FE/BE coupling for an acoustic fluid-structure interaction problem. Residual a posteriori error estimates
}

\author{
* Catalina Domínguez García ${ }^{1}$, Ernst P. Stephan ${ }^{2}$ and Matthias Maischak ${ }^{3}$ \\ 1 Dpto. de Matemáticas y Estadística, Universidad del Norte, Barranquilla, Col. \\ 2 Institut für Angewandte Mathematik, Leibniz Universität Hannover, Hannover, Ger. \\ 3 Department of Mathematical Sciences, Brunel University, London, U.K.
}

\begin{abstract}
SUMMARY
In this paper we develop an a posteriori error analysis of a coupling of finite elements and boundary elements for a fluid-structure interaction problem in two and three dimensions. This problem is governed by the acoustic and the elastodynamic equations in time-harmonic vibration. Our methods combine integral equations for the exterior fluid and finite element methods for the elastic structure. It is well-known that due to the reduction of the boundary value problem to boundary integral equations the solution is not unique in general. However, due to superposition of various potentials, we consider a boundary integral equation which is uniquely solvable and which avoids the irregular frequencies of the negative Laplacian operator of the interior domain. In this paper, two stable procedures are considered; one is based on the non-symmetric formulation and the other one is based on a symmetric formulation. For both formulations we derive reliable residual a posteriori error estimates. From the estimators we compute local error indicators which allow us to develop an adaptive mesh refinement strategy. For the two dimensional case we perform an adaptive algorithm on triangles and for the three dimensional case we use hanging nodes on hexahedrons. Numerical experiments underline our theoretical results. Copyright (c) 2000 John Wiley \& Sons, Ltd.
\end{abstract}

KEY WORDS: Coupling of finite elements and boundary elements, fluid-structure interaction problem, residual a posteriori error estimator, adaptive algorithm.

\section{Introduction}

The problem under consideration consists of determining the dynamics in a fluid $\Omega^{+}$and displacements in an elastic body $\Omega$ due to a given excitation in the fluid $\Omega^{+}$, using an FE/BE coupling method. Here, $\Omega$ is a bounded region in $\mathbb{R}^{d}(d=2,3)$, with boundary $\Gamma$, and $\Omega^{+}:=\mathbb{R}^{d} \backslash \bar{\Omega}$. We consider the scattering of time-harmonic acoustic waves by a bounded elastic obstacle, immersed in a compressible, inviscid, homogeneous fluid. For this type of

\footnotetext{
* Correspondence to: Catalina Domínguez García, Universidad del Norte, Dpto. de Matemáticas y Estadística, Barranquilla, Col. E-mail address: dcatalina@uninorte.edu.co

Contract/grant sponsor: ${ }^{1}$ Deutsche Forschungsgemeinschaft GRK 615, Germany; Universidad del Norte, Barranquilla, Colombia.
} 
problem, the displacement field $\mathbf{u}$ in the domain $\Omega$ and the pressure field $p$ in the fluid are unknown.

In Bielak et al. [1, 2, 3] and Hsiao et al. [4] FE/BE coupling methods for an elastic body are presented to solve the scattering problem, using standard integral representations in the infinite exterior region occupied by the fluid. These methods, however, suffer from the same common defect associated with the integral formulations for purely exterior regions; namely, there is a discrete set of frequencies for which the method fails. Two techniques have been used in applications to remedy this situation. One was developed by Burton and Miller [5], combining linearly the surface Helmholtz integral equation and its normal derivative, derived from Green's second theorem. This method always leads to unique solutions if a certain coupling constant $\alpha$ has a nonvanishing imaginary part. An earlier procedure, given by Brakhage and Werner [6], used far less frequently, represents the solution in the exterior region as a linear combination of a single layer and a double layer potential, with the coupling constant $\alpha$ again required to have a nonvanishing imaginary part. Kress [7] investigated how to choose the parameter $\alpha$ in order to minimize the condition number of the discrete system derived from the integral equation, finding that the value $\alpha=i / k$ is an optimal value, where $k$ is the wavenumber of the acoustic waves in the fluid $\Omega^{+}$.

With respect to the numerical implementation of the $\mathrm{FE} / \mathrm{BE}$ coupling for a fluid-structure interaction problem, we refer to the work of Bielak et al. [2], Chang and Demkowicz [8] and Gatica et al. [9]. Bielak et al. [2] present numerical results for the two dimensional case of a symmetric variational formulation, which is obtained using the procedures of BrakhageWerner and Burton-Miller simultaneously. Chang and Demkowicz present the $h p$-numerical implementation of a variational formulation obtained by the procedure of Burton-Miller and an adaptive $h p$-method based on a residual error estimate that depends only on the pressure in the fluid for a scattering problem in a hollow sphere. Gatica et al. present a mixed finite element method for a fluid-solid interaction problem posed in the plane. Here, a coupling of primal and dual mixed finite element methods is applied to compute both the pressure of the scattered wave in the linearized fluid and the elastic vibrations that take place in the elastic body.

This paper presents the implementation and analysis of a residual a posteriori error estimate of the $\mathrm{FE} / \mathrm{BE}$ coupling methods for two and three dimensional cases and focuses on two stable variational formulations, the symmetric formulation $\left(V P_{1}\right)$ and the non-symmetric formulation $\left(V P_{2}\right)$. With Theorems 4.2 and 4.3 we show reliability of the residual error estimator for formulation $\left(V P_{1}\right)$ and $\left(V P_{2}\right)$ respectively. The efficiency of the error estimator is shown by Theorem I.7 in the Appendix for $\left(V P_{1}\right)$. For $\left(V P_{2}\right)$ it can be shown analogously and is omitted for brevity. We call them stable formulations, because they lead to unique solutions if the coupling constant $\alpha$ has a nonvanishing imaginary part. The non-symmetric formulation stems from the procedure of Brakhage-Werner and the symmetric formulation from using the procedures of Brakhage-Werner and Burton-Miller simultaneously.

The sesquilinear forms corresponding to the variational formulations $\left(V P_{1}\right)$ and $\left(V P_{2}\right)$ are in general not positive definite but satisfy a Gärding's inequality, since they are of the form $(\mathcal{D}+\mathcal{K})$ where $\mathcal{D}$ is a positive definite and $\mathcal{K}$ is a compact sesquilinear form. This allows to apply abstract results for existence and uniqueness of a variational problem, as well as for the stability and convergence analysis of the FE/BE coupling method. The sesquilinear form $\mathcal{D}$ 
induces an energy norm for the problem.

We prove the reliability of a new residual a posteriori error estimate for the stable formulations, which guarantees a quasi-optimal bound of the error in the energy norm induced by $\mathcal{D}$ (Theorems 4.2 and 4.3). Based on these a-posteriori error estimates, we define local indicators and present adaptive algorithms for the mesh refinement of the coupling procedure. The residual error estimates are formulated in the $L^{2}$-norm using standard techniques for FE methods, see e.g. Johnson et al. [10], Stewart et al. [11] and techniques for FE/BE coupling methods e.g. Carstensen and Stephan $[12,13,14]$. To prove its reliability we use arguments of duality, see e.g. Costabel and Stephan [15].

Throughout the rest of the paper we utilize the standard terminology for Sobolev spaces, so $|\cdot|_{r, \Omega}$ and $\|\cdot\|_{r, \Omega}$ stand for the seminorm and norm in the Sobolev spaces $H^{r}(\Omega)$. We write $\|\cdot\|_{m, \Omega}$ instead of $\|\cdot\|_{m}$ whenever the corresponding domain is important to distinguish.

\section{The Fluid-Structure Interaction Problem}

Let $\Omega \subset \mathbb{R}^{d}(d=2,3)$ be a bounded, simply connected domain with a closed polyhedral boundary $\partial \Omega=\Gamma$ and its exterior complement given by $\Omega^{+}:=\mathbb{R}^{d} \backslash \bar{\Omega}$. We assume that all waves are steady-state (time harmonic) with angular frequency $\omega$. If $\Omega$ is a linear elastic body, and/or the solid is subject to a time-harmonic driving force $\mathbf{F}(x, t)=\mathbf{f}(x) e^{-i \omega t}$, the displacement $\mathbf{u}$ is governed by the reduced elastodynamic equation

$$
\operatorname{div} \sigma(\mathbf{u})+\omega^{2} \rho \mathbf{u}=\mathbf{f}
$$

where $\operatorname{div} \sigma(\mathbf{u}):=\mu \Delta \mathbf{u}+(\lambda+\mu) \nabla(\nabla \cdot \mathbf{u})$ is the Lame operator, $\lambda$ and $\mu$ are the Lamé constants and $\rho$ is the density of the body. Let the traction operator $\sigma(\mathbf{u}) \mathbf{n}$ be defined by

$$
\sigma(\mathbf{u}) \mathbf{n}:=2 \mu \frac{\partial \mathbf{u}}{\partial n}+\lambda \mathbf{n} \nabla \cdot \mathbf{u}+\mu \mathbf{n} \times \nabla \mathbf{u} .
$$

$\sigma_{n}$ denotes the normal component of $\sigma(\mathbf{u}) \mathbf{n}$, i.e.,

$$
\sigma_{n}=\mathbf{n}^{T} \sigma(\mathbf{u}) \mathbf{n} .
$$

$\Omega^{+}$represents an inviscid, compressible and homogeneous fluid with density $\rho_{0}$ and speed of sound $c_{0}$. The scalar pressure field in the fluid is denoted by $P(x, t)=p(x) e^{-i \omega t}$. In the fluid $\Omega^{+}$an incident acoustic field $P^{0}(x, t)=p^{0}(x) e^{-i k t}$ is given. The objective is to determine the stationary acoustic field of the scattered pressure $p(x)$ for $x \in \Omega^{+}$, which satisfies the Helmholtz equation

$$
\Delta p+k^{2} p=0,
$$

where $k=\frac{\omega}{c_{0}}$ denotes the wave number, together with the radiation condition

$$
p(x)=\mathcal{O}\left(|x|^{-(d-1) / 2}\right), \quad \frac{d p}{d|x|}(x)-i k p(x)=o\left(|x|^{-(d-1) / 2}\right), \quad|x| \rightarrow \infty .
$$

Moreover the pressure is in static equilibrium with the normal traction on the solid boundary:

$$
\sigma(\mathbf{u}) \mathbf{n}=-\left(p+p^{0}\right) \mathbf{n},
$$


and the normal displacements of the solid and the fluid are equal on the surface. Hence

$$
\rho_{0} \omega^{2} \mathbf{u} \cdot \mathbf{n}=\frac{\partial p}{\partial n}+\frac{\partial p^{0}}{\partial n}
$$

For more details about the governing equations see $[4,16]$. Finally, the fluid-solid interaction problem can be formulated as follows: For a given incident field $p^{0} \in C^{1}$ which satisfies the equation $\Delta p^{0}+k^{2} p^{0}=0$ almost everywhere in $\Omega$ and $\Omega^{+}$, find $\mathbf{u} \in C^{2}(\Omega) \cap C^{1}(\Omega \cup \Gamma)$ and $p \in C^{2}\left(\Omega^{+}\right) \cap C^{1}\left(\Omega^{+} \cup \Gamma\right)$ satisfying

$$
\begin{aligned}
\operatorname{div} \sigma(\mathbf{u})+\rho \omega^{2} \mathbf{u} & =\mathbf{f} \quad \text { in } \Omega, \\
\Delta p+k^{2} p=0 \quad \text { in } \Omega^{+}, & \\
\sigma(\mathbf{u}) \mathbf{n} & =-\left(p+p^{0}\right) \mathbf{n} \quad \text { on } \Gamma \\
\rho_{0} \omega^{2} \mathbf{u} \cdot \mathbf{n} & =\frac{\partial p}{\partial n}+\frac{\partial p^{0}}{\partial n} \quad \text { on } \Gamma
\end{aligned}
$$

$p$ satisfies the radiation condition $(2)$ in $\Omega^{+}$,

where $\mathbf{n}$ is the normal on $\Gamma$ exterior to $\Omega$. The occurrence of resonance frequencies for the interior problem of the Helmholtz equation is typical for problem (3). However we can avoid this phenomenon by taking the representation proposed by Brakhage and Werner in [6], see also $[2,17,7]$. This is formulated as follows: For $\alpha \in \mathbb{C}$ with $\operatorname{Im} \alpha \neq 0$ we use a complex continuous function $\phi$ defined on the boundary $\Gamma$ to represent $p(x)$ by

$$
p(x)=S \phi(x)+\alpha D \phi(x) \quad \forall x \in \Omega^{+},
$$

where $S$ is the single layer potential defined for $x \in \Omega^{+}$by

$$
S(\phi)(x)=\int_{\Gamma} \phi(y) \gamma_{k}(x, y) d s_{y},
$$

$D$ is the double layer potential defined by

$$
D(\phi)(x)=\int_{\Gamma} \phi(y) \frac{\partial \gamma_{k}(x, y)}{\partial n_{y}} d s_{y}
$$

and $\gamma_{k}: \mathbb{R}^{d} \times \mathbb{R}^{d} \rightarrow \mathbb{R}$ is the fundamental solution of the Helmholtz equation given by

$$
\gamma_{k}(x, y):= \begin{cases}\frac{i}{4} H_{0}^{1}(k|x-y|), & \text { for } d=2, \\ \frac{1}{4 \pi} \frac{e^{i k|x-y|}}{|x-y|}, & \text { for } d=3 .\end{cases}
$$

Taking the limit $x \rightarrow \Gamma$ of (5) and (6) and their normal derivatives yields the following jump relations:

$$
\begin{aligned}
S \phi^{ \pm} & =V \phi, & D \phi^{ \pm} & =\left(K \pm \frac{I}{2}\right) \phi, \\
\frac{\partial}{\partial n} S \phi^{ \pm} & =\left(K^{\prime} \mp \frac{I}{2}\right) \phi, & \frac{\partial}{\partial n} D \phi^{ \pm} & =-W \phi,
\end{aligned}
$$


where the upper-indices "-" or "+" indicate from which direction (interior or exterior) the limit is taken and $V, K, K^{\prime}$ and $W$ are the integral operators (see e.g. [18]) defined for $x \in \Gamma$ by

$$
\begin{array}{rlrl}
V(\phi)(x) & :=\int_{\Gamma} \phi(y) \gamma_{k}(x, y) d s_{y}, & K(\phi)(x) & :=\int_{\Gamma} \phi(y) \frac{\partial \gamma_{k}(x, y)}{\partial n_{y}} d s_{y}, \\
W(\phi)(x) & :=-\frac{\partial}{\partial n_{x}} \int_{\Gamma} \phi(y) \frac{\partial}{\partial n_{y}} \gamma_{k}(x, y) d s_{y}, & K^{\prime}(\phi)(x):=\frac{\partial}{\partial n_{x}} \int_{\Gamma} \phi(y) \gamma_{k}(x, y) d s_{y} .
\end{array}
$$

The boundary integral operators $V$ and $\left(K^{\prime}-\frac{I}{2}\right)$ are not invertible if $k^{2}$ is an eigenvalue of the interior Dirichlet Problem for the negative Laplace operator; and $\left(K+\frac{I}{2}\right)$ and $W$ are not invertible if $k^{2}$ is an eigenvalue of the interior Neumann Problem for the negative Laplace operator. This implies that the reduction of boundary value problems to boundary integral equations using these operators is not unique. However, if the representation (4) is used, the corresponding boundary integral equations are uniquely solvable.

Taking the limit $x \rightarrow \Gamma$ from $\Omega^{+}$and applying the jump relations we get that, for all $x \in \Gamma$,

$$
\begin{aligned}
p(x) & =V \phi(x)+\alpha\left(K+\frac{I}{2}\right) \phi(x), \\
\frac{\partial p(x)}{\partial n} & =\left(K^{\prime}-\frac{I}{2}\right) \phi(x)-\alpha W \phi(x) .
\end{aligned}
$$

Inserting the above equalities in the transmission conditions of (3) and using the following notation

$$
\begin{aligned}
a_{0}(\mathbf{u}, \mathbf{v}) & :=\int_{\Omega} \sigma(\mathbf{u}): \operatorname{grad} \overline{\mathbf{v}} d x, & b(\phi, \mathbf{v}) & :=\left\langle\left(V+\left(K+\frac{I}{2}\right)\right)(\phi) \mathbf{n}, \overline{\mathbf{v}}\right\rangle, \\
a_{1}(\mathbf{u}, \mathbf{v}) & :=\int_{\Omega} \mathbf{u} \overline{\mathbf{v}} d x, & b^{\prime}(\mathbf{v}, \phi) & :=\left\langle\left(V+\alpha\left(K^{\prime}+\frac{I}{2}\right)\right)(\mathbf{v}) \cdot \mathbf{n}, \bar{\phi}\right\rangle, \\
a(\mathbf{u}, \mathbf{v}) & :=a_{0}(\mathbf{u}, \mathbf{v})-\rho \omega^{2} a_{1}(\mathbf{u}, \mathbf{v}), & c(\phi, \mathbf{v}) & :=-\langle\phi \mathbf{n}, \overline{\mathbf{v}}\rangle, \\
c^{\prime}(\mathbf{v}, \phi) & :=-\langle\mathbf{v} \cdot \mathbf{n}, \bar{\phi}\rangle & d(\phi, \psi) & :=\frac{1}{\rho_{0} \omega^{2}}\left\langle\left(\left(K^{\prime}-\frac{I}{2}\right)-\alpha W\right) \phi, \bar{\psi}\right\rangle,
\end{aligned}
$$

we can formulate the following stable variational formulations for the problem $(3)$ (see $[2,4])$; the first is a non-symmetric formulation, and the second is a symmetric formulation. In the non-symmetric formulation the unknowns are the displacement $\mathbf{u}$ and the function $\phi$, whereas in the symmetric formulation one considers $\sigma_{n}$ as one additional unknown in the problem. For $\phi \in H^{1 / 2}(\Gamma)$ and $\psi \in H^{-1 / 2}(\Gamma)$ the dual product $\langle\phi, \bar{\psi}\rangle$ is given by $\langle\phi, \bar{\psi}\rangle=\int_{\Gamma} \phi(x) \bar{\psi}(x) d s_{x}$.

Non-symmetric formulation $\left(V P_{1}\right)$. For given $\mathbf{f} \in\left[H^{1}(\Omega)\right]^{d}, p^{0}, \frac{\partial p^{0}}{\partial n} \in H^{1 / 2}(\Gamma)$ find $(\mathbf{u}, \phi) \in \mathcal{H}_{1}:=\left[H^{1}(\Omega)\right]^{d} \times H^{1 / 2}(\Gamma)$ such that

$$
\begin{aligned}
a(\mathbf{u}, \mathbf{v})+b(\phi, \mathbf{v}) & =-(\mathbf{f}, \mathbf{v})_{0}-\left\langle p^{0} \mathbf{n}, \overline{\mathbf{v}}\right\rangle, \\
c(\mathbf{u}, \psi)+d(\phi, \psi) & =-\frac{1}{\rho_{0} \omega^{2}}\left\langle\frac{\partial p^{0}}{\partial n}, \bar{\psi}\right\rangle
\end{aligned} \quad \forall(\mathbf{v}, \psi) \in \mathcal{H}_{1} .
$$

In short: Find $(\mathbf{u}, \phi) \in \mathcal{H}_{1}$ such that

$$
\mathcal{A}_{1}(\mathbf{u}, \phi ; \mathbf{v}, \psi)=\mathcal{F}_{1}(\mathbf{v}, \psi) \quad \forall(\mathbf{v}, \psi) \in \mathcal{H}_{1},
$$


where

$$
\begin{aligned}
\mathcal{A}_{1}(\mathbf{u}, \phi ; \mathbf{v}, \psi) & :=a(\mathbf{u}, \mathbf{v})+b(\phi, \mathbf{v})+c(\mathbf{u}, \psi)+d(\phi, \psi), \\
\mathcal{F}_{1}(\mathbf{v}, \psi) & :=-(\mathbf{f}, \mathbf{v})_{0}-\left\langle p^{0} \mathbf{n}, \overline{\mathbf{v}}\right\rangle-\frac{1}{\rho_{0} \omega^{2}}\left\langle\frac{\partial p^{0}}{\partial n}, \bar{q}\right\rangle
\end{aligned}
$$

Symmetric formulation $\left(V P_{2}\right)$. For given $\mathbf{f} \in\left[H^{1}(\Omega)\right]^{d}, p^{0}, \frac{\partial p^{0}}{\partial n} \in H^{1 / 2}(\Gamma)$ find $\left(\mathbf{u}, \sigma_{n}, \phi\right) \in$ $\mathcal{H}_{2}:=\left[H^{1}(\Omega)\right]^{d} \times H^{1 / 2}(\Gamma) \times H^{1 / 2}(\Gamma)$ such that

$$
\begin{array}{rrrr}
2 a(\mathbf{u}, \mathbf{v})+ & c\left(\sigma_{n}, \mathbf{v}\right)+b(\phi, \mathbf{v}) & =-2(\mathbf{f}, \mathbf{v})_{0}-\left\langle p^{0} \mathbf{n}, \overline{\mathbf{v}}\right\rangle, & \\
c^{\prime}(\mathbf{u}, \chi)+ & +d(\phi, \chi) & =-\frac{1}{\rho_{0} \omega^{2}}\left\langle\frac{\partial p^{0}}{\partial n}, \bar{\chi}\right\rangle, \\
b^{\prime}(\mathbf{u}, \psi)+ & d\left(\bar{\psi}, \overline{\sigma_{n}}\right)+ & & \frac{1}{\rho_{0} \omega^{2}}\left\langle p^{0}+\alpha \frac{\partial p^{0}}{\partial n}, \bar{\psi}\right\rangle
\end{array} \quad \forall(\mathbf{v}, \chi, \psi) \in \mathcal{H}_{2} .
$$

In short: Find $\left(\mathbf{u}, \sigma_{n}, \phi\right) \in \mathcal{H}_{2}$ such that

$$
\mathcal{A}_{2}\left(\mathbf{u}, \sigma_{n}, \phi ; \mathbf{v}, \chi, \psi\right)=\mathcal{F}_{2}(\mathbf{v}, \chi, \psi) \quad \forall(\mathbf{v}, \chi, \psi) \in \mathcal{H}_{2},
$$

where

$$
\begin{aligned}
\mathcal{A}_{2}\left(\mathbf{u}, \sigma_{n}, \phi ; \mathbf{v}, \chi, \psi\right):= & 2 a(\mathbf{u}, \mathbf{v})+b(\phi, \mathbf{v})+b^{\prime}(\mathbf{u}, \psi) \\
& +c\left(\sigma_{n}, \overline{\mathbf{v}}\right)+c^{\prime}(\mathbf{u}, \chi)+d(\phi, \chi)+d\left(\bar{\psi}, \overline{\sigma_{n}}\right) \\
\mathcal{F}_{2}(\mathbf{v}, \chi, \psi):= & -2(\mathbf{f}, \mathbf{v})_{0}-\left\langle p^{0} \mathbf{n}, \overline{\mathbf{v}}\right\rangle-\left\langle\frac{\partial p^{0}}{\partial n}, \bar{\chi}\right\rangle+\frac{1}{\rho_{0} \omega^{2}}\left\langle p^{0}+\alpha \frac{\partial p^{0}}{\partial n}, \bar{\psi}\right\rangle .
\end{aligned}
$$

The above variational formulations are in general not positive definite, here the sesquilinear forms $\mathcal{A}(\cdot, \cdot)$ are of the form $\mathcal{A}=\mathcal{D}+\mathcal{K}$ where $\mathcal{D}$ is positive definite and $\mathcal{K}$ is compact. Then, the existence of a unique solution of the weak formulations $\left(V P_{1}\right)$ and $\left(V P_{2}\right)$ can be concluded from the fact that these satisfy a Gårding's inequality. This type of problem satisfies the Fredholm alternative: either the variational problem has a unique solution or there exists a nontrivial solution of the homogeneous problem. Hence the existence of the solution follows if one can show uniqueness.

Proposition 2.1 ([1]) Given $\mathbf{f} \in\left[H^{1}(\Omega)\right]^{d}, p^{0} \in H^{1 / 2}(\Omega), \frac{\partial p^{0}}{\partial n} \in H^{-1 / 2}(\Omega)$, if $\alpha \in \mathbb{C}$ and $\alpha \neq 0$ then for every $k \in \mathbb{R}$ the formulations $\left(V P_{1}\right)$ and $\left(V P_{2}\right)$ have a unique solution $(\mathbf{u}, \phi) \in \mathcal{H}_{1}$ and $\left(\mathbf{u}, \sigma_{n}, \phi\right) \in \mathcal{H}_{2}$ respectively.

\section{FE/BE Coupling Method}

In order to derive the a posteriori residual error estimate we consider a regular decomposition $\mathcal{T}_{h}$ of $\Omega \subset \mathbb{R}^{d} d=2,3$ into non-overlapping elements $\tau$ of diameter $h_{\tau}$, where $h:=\max _{\tau \in \mathcal{T}_{h}} h_{\tau}$. We assume that $\mathcal{T}_{h}$ is quasi-uniform with mesh size $h>0$ and shape-regular. Let $\mathcal{S}_{\Gamma, \tilde{h}}$ the set of faces $s$ of elements $\tau \in \mathcal{T}_{h}$ which are contained in $\Gamma$ with mesh size $\tilde{h}$. We also assume that $\mathcal{S}_{\Gamma, \tilde{h}}$ is a regular mesh. Let $\mathcal{P}_{1}$ denote the set of polynomials of degree $\leq 1$. Let $\mathcal{W}^{h} \subset H^{1}(\Omega)$ be the space of continuous and piecewise polynomials with respect to a decomposition of $\Omega$ defined by

$$
\mathcal{W}^{h}:=\left\{\eta \in C^{0}(\Omega): \eta_{\mid \tau} \in \mathcal{P}_{1} \text { for every } \tau \in \mathcal{T}_{h}\right\}
$$


and let $\mathcal{B}^{h}$ be the vector space of continuous and piecewise polynomials with respect to a decomposition of the boundary $\Gamma$ defined by

$$
\mathcal{B}^{h}:=\left\{\eta \in C^{0}(\Gamma): \eta_{\mid s} \in \mathcal{P}_{1} \text { for every } s \in \mathcal{S}_{\Gamma, \tilde{h}}\right\}
$$

Let us define the following spaces that correspond to the formulations $\left(V P_{1}\right)$ and $\left(V P_{2}\right)$,

$$
\mathcal{H}_{1}^{h}:=\left[\mathcal{W}^{h}\right]^{d} \times \mathcal{B}^{h}, \quad \mathcal{H}_{2}^{h}:=\left[\mathcal{W}^{h}\right]^{d} \times \mathcal{B}^{h} \times \mathcal{B}^{h} .
$$

We get the following discrete formulations of $\left(V P_{1}\right)$ and $\left(V P_{2}\right)$, respectively: Find $\left(\mathbf{u}^{h}, \phi^{h}\right) \in$ $\mathcal{H}_{1}^{h}$ such that

$$
\mathcal{A}_{1}\left(\mathbf{u}^{h}, \phi^{h} ; \mathbf{v}^{h}, \psi^{h}\right)=\mathcal{F}_{1}\left(\mathbf{v}^{h}, \psi^{h}\right) \quad \forall\left(\mathbf{v}^{h}, \psi^{h}\right) \in \mathcal{H}_{1}^{h} .
$$

Find $\left(\mathbf{u}^{h}, \sigma_{n}^{h}, \phi^{h}\right) \in \mathcal{H}_{2}^{h}$ such that

$$
\mathcal{A}_{2}\left(\mathbf{u}^{h}, \sigma_{n}^{h}, \phi^{h} ; \mathbf{v}^{h}, \chi^{h}, \psi^{h}\right)=\mathcal{F}_{2}\left(\mathbf{v}^{h}, \chi^{h}, \psi^{h}\right) \quad\left(\mathbf{v}^{h}, \chi^{h}, \psi^{h}\right) \in \mathcal{H}_{2}^{h} .
$$

The proof of uniqueness and convergence of the solution of the discrete variational problems $\left(V P_{1}^{h}\right)$ and $\left(V P_{2}^{h}\right)$ can be found in $[19,1]$.

Proposition 3.1. Let $(\mathbf{u}, \phi)$ solve $\left(V P_{1}\right)$. Then there exist $h_{0}>0$ and $c>0$ such that for any $h<h_{0}\left(V P_{1}^{h}\right)$ has a unique solution $\left(\mathbf{u}^{h}, \phi^{h}\right) \in \mathcal{H}_{1}^{h}$ and

$$
\left\|(\mathbf{u}, \phi)-\left(\mathbf{u}^{h}, \phi^{h}\right)\right\|_{\mathcal{H}_{1}} \leq c\left(\inf _{v^{h} \in\left[W^{h}(\Omega)\right]^{d}}\left\|\mathbf{u}-\mathbf{v}^{h}\right\|_{1}^{2}+\inf _{\psi^{h} \in S^{h}}\left\|\phi-\psi^{h}\right\|_{1 / 2}^{2}\right)^{1 / 2} .
$$

Let $\left(\mathbf{u}, \sigma_{n}, \phi\right)$ solve $\left(V P_{2}\right)$. Then there exists $h_{0}>0$ and $c>0$ such that for any $h<h_{0}\left(V P_{2}^{h}\right)$ has a unique solution $\left(\mathbf{u}^{h}, \sigma_{n}^{h}, \phi^{h}\right) \in \mathcal{H}_{2}^{h}$ and

$$
\begin{aligned}
\left\|\left(\mathbf{u}, \sigma_{n}, \phi\right)-\left(\mathbf{u}^{h}, \sigma_{n}^{h}, \phi^{h}\right)\right\|_{\mathcal{H}_{2}} \leq c\left(\inf _{v^{h} \in\left[W^{h}(\Omega)\right]^{d}}\left\|\mathbf{u}-\mathbf{v}^{h}\right\|_{1}^{2}\right. & \\
& \left.+\inf _{\psi^{h} \in S h}\left\|\sigma_{n}-\chi^{h}\right\|_{1 / 2}^{2}+\inf _{\chi^{h} \in S h}\left\|\phi-\psi^{h}\right\|_{1 / 2}^{2}\right)^{1 / 2} .
\end{aligned}
$$

\section{Residual A Posteriori Error Estimate}

We present an a posteriori residual error estimator for the formulations $\left(V P_{1}\right)$ and $\left(V P_{2}\right)$. The residual error estimate is formulated in the $L^{2}$-norm using standard techniques for $\mathrm{FE}$ methods, see [11] and techniques for FE/BE coupling methods e.g. [20, 12, 13, 14]. The derived error indicators are used later for the implementation of adaptive algorithms. First we present the analysis for the non symmetric formulation $\left(V P_{1}\right)$.

Let $\mathcal{S}_{i}$ denote the set of faces of $\mathcal{T}_{h}$ which are not contained in $\mathcal{S}_{\Gamma, \tilde{h}}$. For a element $\tau \in \mathcal{T}_{h}$ of length $h_{\tau}$, we define the set of interior faces of $\tau$ by $\mathcal{S}_{\tau, i}$ which are not contained in $\mathcal{S}_{\Gamma, \tilde{h}}$ and the boundary faces of $\tau$ contained in $\mathcal{S}_{\Gamma, \tilde{h}}$ by $\mathcal{S}_{\tau, \Gamma}$. Note that the set of faces of $\tau$ is given by $\mathcal{S}_{\tau, i} \cup \mathcal{S}_{\tau, \Gamma}=\mathcal{S}_{\tau}$. In the following we denote the space of continuous and piecewise polynomials on $\tau$ and on the face $s$ by $\mathcal{W}^{h}(\tau)$ and $\mathcal{B}^{h}(s)$, respectively.

Then for the Clement approximation operator $\mathcal{I}$ hold the following approximate properties. There exist positive constants $c_{1}, c_{2}$ independent of $\tau \in \mathcal{T}_{h}$ and $h$, such that for every $\eta \in H^{1}(\tau)$ there exists $\mathcal{I} \eta \in \mathcal{W}^{h}(\tau)$ such that

$$
\|\eta-\mathcal{I} \eta\|_{0, \tau} \leq c_{1} h|\eta|_{1, \tau}
$$


and

$$
\|\eta-\mathcal{I} \eta\|_{0, \partial \tau} \leq c_{2} h^{1 / 2}|\eta|_{1, \tau}
$$

There also exists a constant $c_{3}>0$ independent of $s \in \mathcal{S}_{\Gamma, \tilde{h}}$ and $\tilde{h}$, such that for every $\zeta \in H^{1 / 2}(s)$ there exists $\mathcal{I} \zeta \in \mathcal{B}^{h}(s)$ such that

$$
\|\zeta-\mathcal{I} \zeta\|_{0, s} \leq c_{3} \tilde{h}^{1 / 2}\|\zeta\|_{\frac{1}{2}, s} .
$$

Remark 4.1. Since the sesquilinear form $\mathcal{A}_{1}$ is self-adjoint except in terms which contain the operator $K$, whose adjoint is $K^{\prime}$, we can apply the same uniqueness and existence theory applied for the formulation $\mathcal{A}_{1}^{*}$ and obtain that there exists a unique solution $(\mathbf{u}, \phi) \in \mathcal{H}$ of the following adjoint variational problem

$$
\mathcal{A}_{1}^{*}(\mathbf{v}, \psi, \mathbf{u}, \phi):=\mathcal{A}_{1}(\overline{\mathbf{v}, \psi} ; \overline{\mathbf{u}, \phi})=\mathcal{F}_{1}(\mathbf{v}, \psi) \quad \forall(\mathbf{v}, \psi) \in \mathcal{H}_{1} .
$$

Let $\mathcal{H}_{1}^{\prime}=H^{-1}(\Omega) \times H^{-1 / 2}(\Gamma)$. From the above statements we obtain that there are continuous and invertible operators $A_{1}: \mathcal{H}_{1} \rightarrow \mathcal{H}_{1}^{\prime}$ and $A_{1}^{*}: \mathcal{H}_{1} \rightarrow \mathcal{H}_{1}^{\prime}$ such that

$$
\mathcal{A}_{1}(\eta, \zeta)=\left\langle A_{1} \eta, \zeta\right\rangle_{\mathcal{H}_{1}^{\prime}}, \quad \mathcal{A}_{1}^{*}(\zeta, \eta)=\left\langle A_{1}^{*} \zeta, \eta\right\rangle_{\mathcal{H}_{1}^{\prime}},
$$

for all $(\eta, \zeta) \in \mathcal{H}_{1} \times \mathcal{H}_{1}$.

Theorem 4.2. Let $(\mathbf{u}, \phi) \in \mathcal{H}_{1}$ be the solution of problem $\left(V P_{1}\right)$ and $\left(\mathbf{u}^{h}, \phi^{h}\right) \in \mathcal{H}_{1}^{h}$ be the solution of the discrete problem $\left(V P_{1}^{h}\right)$. There exists a positive constant $c$ independent of the meshsize $h$ such that

$$
\left\|\mathbf{u}^{h}-\mathbf{u}, \phi^{h}-\phi\right\|_{\mathcal{H}_{1}} \leq c\left(R_{1}^{h}+R_{2}^{h}+R_{3}^{h}+R_{4}^{h}\right)^{1 / 2},
$$

where

$$
\begin{aligned}
& R_{1}^{h}:=\sum_{\tau \in \mathcal{T}_{h}} h_{\tau}^{2}\left\|\operatorname{div} \sigma\left(\mathbf{u}^{h}\right)+\rho \omega^{2} \mathbf{u}^{h}-\mathbf{f}\right\|_{0, \tau}^{2}, \\
& R_{2}^{h}:=\sum_{s_{i} \in \mathcal{S}_{i}} h_{s_{i}}\left\|\llbracket \sigma\left(\mathbf{u}^{h}\right) \cdot \mathbf{n} \rrbracket\right\|_{0, s_{i}}^{2}, \\
& R_{3}^{h}:=\sum_{s \in \mathcal{S}_{\Gamma, \tilde{h}}} h_{s}\left\|\sigma\left(\mathbf{u}^{h}\right) \cdot \mathbf{n}+p^{0} \mathbf{n}+V \phi^{h} \mathbf{n}+\alpha\left(K+\frac{I}{2}\right) \phi^{h} \mathbf{n}\right\|_{0, s}^{2}, \\
& R_{4}^{h}:=\sum_{s \in \mathcal{S}_{\Gamma, \tilde{h}}} h_{s} \|-\frac{1}{\rho_{0} \omega^{2}} \frac{\partial p^{0}}{\partial n}+\mathbf{u}^{h} \cdot \mathbf{n}-\frac{1}{\rho_{0} \omega^{2}}\left(\left(\left(K^{\prime}-\frac{I}{2}\right)-\alpha W\right) \phi^{h} \|_{0, s}^{2} .\right.
\end{aligned}
$$

Proof. First, we apply a duality argument to obtain (16). This argument of duality is necessary because our bilinear form $\mathcal{A}_{1}$ is not coercive, and therefore it is impossible to use the orthogonality property of the energy norm to bound the error $e$ by the residual of the approximate solution $\left(\mathbf{u}^{h}, \phi^{h}\right)$.

Let $e:=\left(\mathbf{u}-\mathbf{u}^{h}, \phi-\phi^{h}\right)$. From Remark 4.1 it follows that the adjoint equation $A_{1}^{*} \delta=\eta$ is uniquely solvable for every $\eta \in \mathcal{H}_{1}^{\prime}$. Moreover the continuity of $\left(A_{1}^{*}\right)^{-1}: \mathcal{H}_{1}^{\prime} \rightarrow \mathcal{H}_{1}$ implies

$$
\|\delta\|_{\mathcal{H}_{1}} \leq c\|\eta\|_{\mathcal{H}_{1}^{\prime}} \quad \forall(\delta, \eta) \in \mathcal{H}_{1} \times \mathcal{H}_{1}^{\prime} .
$$


Since $\mathcal{H}_{1}$ and $\mathcal{H}_{1}^{\prime}$ are dual with respect to the scalar product of $\mathcal{L}^{2}:=\left[L^{2}(\Omega)\right]^{d} \times L^{2}(\Gamma)$, we have

$$
\|e\|_{\mathcal{H}_{1}} \leq \sup _{\|\eta\|_{\mathcal{H}_{1}^{\prime}} \leq 1}\left|(e, \eta)_{\mathcal{L}^{2}}\right|=\sup _{\left\|A^{*} \delta\right\|_{\mathcal{H}_{1}^{\prime}} \leq 1}\left|\left(e, A_{1}^{*} \delta\right)_{\mathcal{L}^{2}}\right|=\sup _{\left\|A^{*} \delta\right\|_{\mathcal{H}_{1}^{\prime}} \leq 1}\left|\left(A_{1} e, \delta\right)_{\mathcal{L}^{2}}\right|
$$

Now, we associate the term $\left(A_{1} e, \delta\right)$ with the residual of the approximate solution $\left(\mathbf{u}^{h}, \phi^{h}\right)$.

$$
\left(A_{1} e, \delta\right)=\mathcal{A}_{1}(e ; \delta)=\mathcal{A}_{1}\left(\mathbf{u}^{h}, \phi^{h} ; \delta\right)-\mathcal{A}_{1}(\mathbf{u}, \phi ; \delta)=\mathcal{A}_{1}\left(\mathbf{u}^{h}, \phi^{h} ; \delta\right)-\mathcal{F}_{1}(\delta)
$$

For $l \in \mathcal{H}_{1}^{h}$ there holds $\mathcal{A}_{1}\left(\mathbf{u}^{h}, \phi^{h} ; l\right)-\mathcal{F}_{1}(l)=0$. Then

$$
\begin{aligned}
\mathcal{A}_{1}(e ; \delta) & =\mathcal{A}_{1}\left(\mathbf{u}^{h}, \phi^{h} ; \delta\right)-\mathcal{F}_{1}(\delta)+\mathcal{F}_{1}(l)-\mathcal{A}_{1}\left(\mathbf{u}^{h}, \phi^{h} ; l\right) \\
& =\mathcal{F}_{1}(l-\delta)-\mathcal{A}_{1}\left(\mathbf{u}^{h}, \phi^{h} ; l-\delta\right) .
\end{aligned}
$$

Taking $l$ as the $L^{2}$-projection of $\delta$ on $\mathcal{H}_{1}^{h}$ and $\eta:=\left(\eta_{\mathbf{v}}, \eta_{\psi}\right)=l-\delta \in \mathcal{H}_{1}$, we get

$$
\mathcal{A}_{1}(e ; \delta)=\mathcal{F}_{1}(\eta)-\mathcal{A}_{1}\left(\mathbf{u}^{h}, \phi^{h} ; \eta\right) .
$$

We use (17) to obtain our error estimator by estimating the residual on every element and on every face. Applying integration by parts to $a_{0}\left(\mathbf{u}^{h}, \eta_{\mathbf{v}}\right)_{\tau}$ for $\tau \in \mathcal{T}_{h}$ we obtain

$$
\begin{aligned}
a_{0}\left(\mathbf{u}^{h}, \eta_{\mathbf{v}}\right)_{\tau} & =\left(\sigma\left(\mathbf{u}^{h}\right): \nabla \eta_{\mathbf{v}}\right)_{0, \tau}=-\left(\operatorname{div} \sigma\left(\mathbf{u}^{h}\right), \eta_{\mathbf{v}}\right)_{0, \tau}+\int_{\partial \tau}\left(\sigma\left(\mathbf{u}^{h}\right) \mathbf{n}\right) \cdot \bar{\eta}_{\mathbf{v}} d s \\
& \left.=-\left(\operatorname{div} \sigma\left(\mathbf{u}^{h}\right), \eta_{\mathbf{v}}\right)_{0, \tau}+\sum_{s_{i} \in \mathcal{S}_{\tau, i}}\left\langle\sigma\left(\mathbf{u}^{h}\right) \cdot \mathbf{n}, \bar{\eta}_{\mathbf{v}}\right\rangle_{0, s_{i}}+\sum_{s \in \mathcal{S}_{\tau, \Gamma}}\left\langle\sigma\left(\mathbf{u}^{h}\right) \cdot \mathbf{n}\right), \bar{\eta}_{\mathbf{v}}\right\rangle_{0, s} \\
& =-\left(\operatorname{div} \sigma\left(\mathbf{u}^{h}\right), \eta_{\mathbf{v}}\right)_{0, \tau}+\sum_{s_{i} \in \mathcal{S}_{\tau, i}}\left\langle\sigma\left(\mathbf{u}^{h}\right) \cdot \mathbf{n}, \bar{\eta}_{\mathbf{v}}\right\rangle_{0, s_{i}}+\left\langle\sigma\left(\mathbf{u}^{h}\right) \mathbf{n}, \bar{\eta}_{\mathbf{v}}\right\rangle_{0} .
\end{aligned}
$$

Note that the resulting boundary integral over $\partial \tau$ is decomposed into integrals on each interior face and on each boundary face. Inserting (9) and (18) into (17) we get

$$
\begin{aligned}
\mathcal{A}_{1}(e ; \delta)= & \mathcal{F}_{1}(\eta)-\mathcal{A}_{1}\left(\mathbf{u}^{h}, \phi^{h} ; \eta\right) \\
= & \sum_{\tau \in \mathcal{T}_{h}}\left(\left(\operatorname{div} \sigma\left(\mathbf{u}^{h}\right), \eta_{\mathbf{v}}\right)_{0, \tau}+\rho \omega^{2}\left(\mathbf{u}^{h}, \eta_{\mathbf{v}}\right)_{0, \tau}-\left(\mathbf{f}, \eta_{\mathbf{v}}\right)_{0, \tau}\right) \\
& -\sum_{s_{i} \in \mathcal{S}_{i}}\left\langle\llbracket \sigma\left(\mathbf{u}^{h}\right) \mathbf{n} \rrbracket, \bar{\eta}_{\mathbf{v}}\right\rangle_{0, s_{i}}-\left\langle\sigma\left(\mathbf{u}^{h}\right) \mathbf{n}, \bar{\eta}_{\mathbf{v}}\right\rangle_{0} \\
& -\left\langle p^{0} \mathbf{n}, \bar{\eta}_{\mathbf{v}}\right\rangle_{0}-\frac{1}{\rho_{0} \omega^{2}}\left\langle\frac{\partial p^{0}}{\partial n}, \bar{\eta}_{\psi}\right\rangle_{0}-\left\langle\left(V-\alpha\left(K+\frac{I}{2}\right)\right) \phi^{h} \mathbf{n}, \bar{\eta}_{\mathbf{v}}\right\rangle_{0} \\
& +\left\langle\mathbf{u}^{h} \cdot \mathbf{n}, \bar{\eta}_{\psi}\right\rangle_{0}-\frac{1}{\rho_{0} \omega^{2}}\left\langle\left(\left(K^{\prime}-\frac{I}{2}\right)-\alpha W\right) \phi^{h}, \bar{\eta}_{\psi}\right\rangle_{0} \\
= & \sum_{\tau \in \mathcal{T}_{h}}\left(r_{1}^{h}, \eta_{\mathbf{v}}\right)_{\tau}+\left(r_{2}^{h}, \bar{\eta}\right),
\end{aligned}
$$

where $\llbracket \cdot \rrbracket$ denotes the jump over an interior face, $r_{1}^{h}:=\operatorname{div} \sigma\left(\mathbf{u}^{h}\right)+\rho \omega^{2} \mathbf{u}^{h}-\mathbf{f}$ is the residual defined on the interior elements, and $\left(r_{2}^{h}, \bar{\eta}\right)$ denotes the residuals defined on the boundary 
elements. Using the Cauchy-Schwarz inequality, (10)-(12) and the Hölder inequality we obtain an upper bound of (19) in terms of our error estimator

$$
\begin{aligned}
& \left|\sum_{\tau \in \mathcal{T}_{h}}\left(r_{1}^{h}, \eta_{\mathbf{v}}\right)_{\tau}+\left(r_{2}^{h}, \bar{\eta}\right)\right| \\
& \left.\leq \sum_{\tau \in \mathcal{T}_{h}}\left\|r_{1}^{h}\right\|_{0, \tau}\left\|\eta_{\mathbf{v}}\right\|_{0, \tau}+\sum_{s_{i} \in \mathcal{S}_{i}} \| \llbracket \sigma\left(\mathbf{u}^{h}\right) \mathbf{n}\right) \rrbracket\left\|_{0, s_{i}}\right\| \eta_{\mathbf{v}} \|_{0, s_{i}} \\
& +\sum_{s \in \mathcal{S}_{\Gamma, \tilde{h}}}\left\|-\sigma\left(\mathbf{u}^{h}\right) \mathbf{n}-p^{0} \mathbf{n}-V \phi^{h} \mathbf{n}-\alpha\left(K+\frac{I}{2}\right) \phi^{h} \mathbf{n}\right\|_{0, s}\left\|\eta_{\mathbf{v}}\right\|_{0, s} \\
& +\sum_{s \in \mathcal{S}_{\Gamma, \tilde{h}}}\left\|-\frac{1}{\rho_{0} \omega^{2}} \frac{\partial p^{0}}{\partial n}+\mathbf{u}^{h} \cdot \mathbf{n}-\frac{1}{\rho_{0} \omega^{2}}\left(\left(K^{\prime}-\frac{I}{2}\right)-\alpha W\right) \phi^{h}\right\|_{0, s}\left\|\eta_{\psi}\right\|_{0, s} \\
& \left.\leq c_{1} \sum_{\tau \in \mathcal{T}_{h}} h_{\tau}\left\|r_{1}^{h}\right\|_{0, \tau}\left|\delta_{\mathbf{v}}\right|_{1, \tau}+c_{2} \sum_{s_{i} \in \mathcal{S}_{i}} \| \llbracket \sigma\left(\mathbf{u}^{h}\right) \mathbf{n}\right) \rrbracket \|_{0, s_{i}} h_{s_{i}}^{1 / 2}\left|\delta_{\mathbf{v}}\right|_{1 / 2, s_{i}} \\
& +c_{2} \sum_{s \in \mathcal{S}_{\Gamma, \tilde{h}}}\left\|\sigma\left(\mathbf{u}^{h}\right) \mathbf{n}+p^{0} \mathbf{n}+V \phi^{h} \mathbf{n}+\alpha\left(K+\frac{I}{2}\right) \phi^{h} \mathbf{n}\right\|_{0, s} h_{s}^{1 / 2}\left|\delta_{\mathbf{v}}\right|_{1 / 2, s} \\
& +c_{3} \sum_{s \in \mathcal{S}_{\Gamma, \tilde{h}}}\left\|-\frac{1}{\rho_{0} \omega^{2}} \frac{\partial p^{0}}{\partial n}+\mathbf{u}^{h} \cdot \mathbf{n}-\frac{1}{\rho_{0} \omega^{2}}\left(\left(K^{\prime}-\frac{I}{2}\right)-\alpha W\right) \phi^{h}\right\|_{0, s} h_{s}^{\frac{1}{2}}\left\|\delta_{\psi}\right\|_{\frac{1}{2}, s} \\
& \leq \max \left\{c_{1}, c_{2}, c_{3}\right\}\left(\sum_{\tau \in \mathcal{T}_{h}} h_{\tau}^{2}\left\|r_{1}^{h}\right\|_{0, \tau}^{2}+\sum_{s_{i} \in \mathcal{S}_{i}} h_{s_{i}}\left\|\llbracket \sigma\left(\mathbf{u}^{h}\right) \mathbf{n} \rrbracket\right\|_{0, s_{i}}^{2}\right. \\
& +\sum_{s \in \mathcal{S}_{\Gamma, \tilde{h}}} h_{s}\left\|\sigma\left(\mathbf{u}^{h}\right) \mathbf{n}+p^{0} \mathbf{n}+V \phi^{h} \mathbf{n}+\alpha\left(K+\frac{I}{2}\right) \phi^{h} \mathbf{n}\right\|_{0, s}^{2} \\
& \left.+\sum_{s \in \mathcal{S}_{\Gamma, \tilde{h}}} h_{s}\left\|-\frac{1}{\rho_{0} \omega^{2}} \frac{\partial p^{0}}{\partial n}+\mathbf{u}^{h} \cdot \mathbf{n}-\frac{1}{\rho_{0} \omega^{2}}\left(\left(K^{\prime}-\frac{I}{2}\right)-\alpha W\right) \phi^{h}\right\|_{0, s}^{2}\right)^{1 / 2} \\
& \times\left(\sum_{\tau \in \mathcal{T}_{h}}\left|\delta_{\mathbf{v}}\right|_{1, \tau}^{2}+\sum_{\tau \in \mathcal{T}_{h}}\left|\delta_{\mathbf{v}}\right|_{1, s_{i}}^{2}+\sum_{s \in \mathcal{S}_{\Gamma, \tilde{h}}}\left|\delta_{\mathbf{v}}\right|_{1, s}^{2}+\sum_{s \in \mathcal{S}_{\Gamma, \tilde{h}}}\left\|\delta_{\psi}\right\|_{1 / 2, s}^{2}\right)^{1 / 2} \\
& \leq \max \left\{c_{1}, c_{2}, c_{3}\right\}\left(R_{1}^{h}+R_{2}^{h}+R_{3}^{h}+R_{4}^{h}\right)^{1 / 2} \\
& \times\left(\sum_{\tau \in \mathcal{T}_{h}}\left\|\delta_{\mathbf{v}}\right\|_{1, \tau}^{2}+\sum_{s \in \mathcal{S}_{\Gamma, \tilde{h}}}\left\|\delta_{\psi}\right\|_{1 / 2, s}^{2}\right)^{1 / 2} \\
& \leq \max \left\{c_{1}, c_{2}, c_{3}\right\}\left(R_{1}^{h}+R_{2}^{h}+R_{3}^{h}+R_{4}^{h}\right)^{1 / 2}\|\delta\|_{\mathcal{H}^{1}} .
\end{aligned}
$$

Finally, starting from (16), using (15), (19) and the above inequality, we get

$$
\begin{aligned}
\left\|\mathbf{u}^{h}-\mathbf{u}, \phi^{h}-\phi\right\|_{\mathcal{H}^{1}} & \leq \max \left\{c_{1}, c_{2}, c_{3}\right\}\left(R_{1}^{h}+R_{2}^{h}+R_{3}^{h}+R_{4}^{h}\right)^{1 / 2}\|\delta\|_{\mathcal{H}^{1}} \\
& \leq \max \left\{c_{1}, c_{2}, c_{3}\right\}\left(R_{1}^{h}+R_{2}^{h}+R_{3}^{h}+R_{4}^{h}\right)^{1 / 2} .
\end{aligned}
$$

The Appendix shows the demonstration of efficiency of error estimator (13) for the formulation $\left(V P_{1}\right)$. Now we formulate the error estimator for the symmetric formulation $\left(V P_{2}\right)$. 
Theorem 4.3. Let $\left(\mathbf{u}, \sigma_{n}, \phi\right) \in \mathcal{H}_{2}$ be the solution of problem $\left(V P_{2}\right)$ and let $\left(\mathbf{u}^{h}, \sigma_{n}^{h}, \phi^{h}\right) \in \mathcal{H}_{2}^{h}$ be the solution of the discrete problem $\left(V P_{2}^{h}\right)$. There exists a positive constant $c$ such that

$$
\left\|\mathbf{u}^{h}-\mathbf{u}, \sigma_{n}^{h}-\sigma_{n}, \phi^{h}-\phi\right\|_{\mathcal{H}_{2}} \leq c\left(\tilde{R}_{1}^{h}+\tilde{R}_{2}^{h}+\tilde{R}_{3}^{h}+\tilde{R}_{4}^{h}+\tilde{R}_{5}^{h}\right)^{1 / 2}
$$

where

$$
\begin{aligned}
& \tilde{R}_{1}^{h}:=\sum_{\tau \in \mathcal{T}_{h}} 2 h_{\tau}^{2}\left\|\operatorname{div} \sigma\left(\mathbf{u}^{h}\right)+\rho \omega^{2} \mathbf{u}^{h}-\mathbf{f}\right\|_{0, \tau}^{2}, \\
& \tilde{R}_{2}^{h}:=\sum_{s_{i} \in \mathcal{S}_{i}} 2 h_{s_{i}}\left\|\llbracket \sigma\left(\mathbf{u}^{h}\right) \mathbf{n} \rrbracket\right\|_{s_{i}}^{2}, \\
& \tilde{R}_{3}^{h}:=\sum_{s \in \mathcal{S}_{\Gamma, \tilde{h}}} h_{s}\left\|-2 \sigma\left(\mathbf{u}^{h}\right) \mathbf{n}-p^{0} \mathbf{n}+\sigma_{n}^{h} \mathbf{n}-\left(V+\alpha\left(K+\frac{I}{2}\right)\right) \phi^{h} \mathbf{n}\right\|_{s}^{2}, \\
& \tilde{R}_{4}^{h}:=\sum_{s \in \mathcal{S}_{\Gamma, \tilde{h}}} h_{s}\left\|-\frac{1}{\rho_{0} \omega^{2}} \frac{\partial p^{0}}{\partial n}+\mathbf{u}^{h} \cdot \mathbf{n}-\frac{1}{\rho_{0} \omega^{2}}\left(\left(K^{\prime}-\frac{I}{2}\right)-\alpha W\right) \phi^{h}\right\|_{s}^{2}, \\
& \tilde{R}_{5}^{h}:=\sum_{s \in \mathcal{S}_{\Gamma, \tilde{h}}} h_{s} \| \frac{1}{\rho_{0} \omega^{2}}\left(p^{0}+\alpha \frac{\partial p^{0}}{\partial n}\right)-\left(V+\alpha\left(K^{\prime}+\frac{I}{2}\right)\right) \mathbf{u}^{h} \cdot \mathbf{n} \\
& \quad-\frac{1}{\rho_{0} \omega^{2}}\left(\left(K-\frac{I}{2}\right)-\alpha W\right) \sigma_{n}^{h} \|_{s}^{2} .
\end{aligned}
$$

Proof. The proof is analogous to the one given in Theorem 4.2 and is therefore omitted for brevity.

In Algorithm 1 we compute the local error indicators by restricting the estimates $\eta_{R_{1}}:=$ $\left(R_{1}^{h}+R_{2}^{h}+R_{3}^{h}+R_{4}^{h}\right)^{1 / 2}$ and $\eta_{R_{2}}:=\left(\tilde{R}_{1}^{h}+\tilde{R}_{2}^{h}+\tilde{R}_{3}^{h}+\tilde{R}_{4}^{h}+\tilde{R}_{5}^{h}\right)^{1 / 2}$ to an element $\tau \in \mathcal{T}_{h}$. We denote these local error indicator as $\eta_{R_{1}}^{\tau}$ and $\eta_{R_{2}}^{\tau}$, respectively.

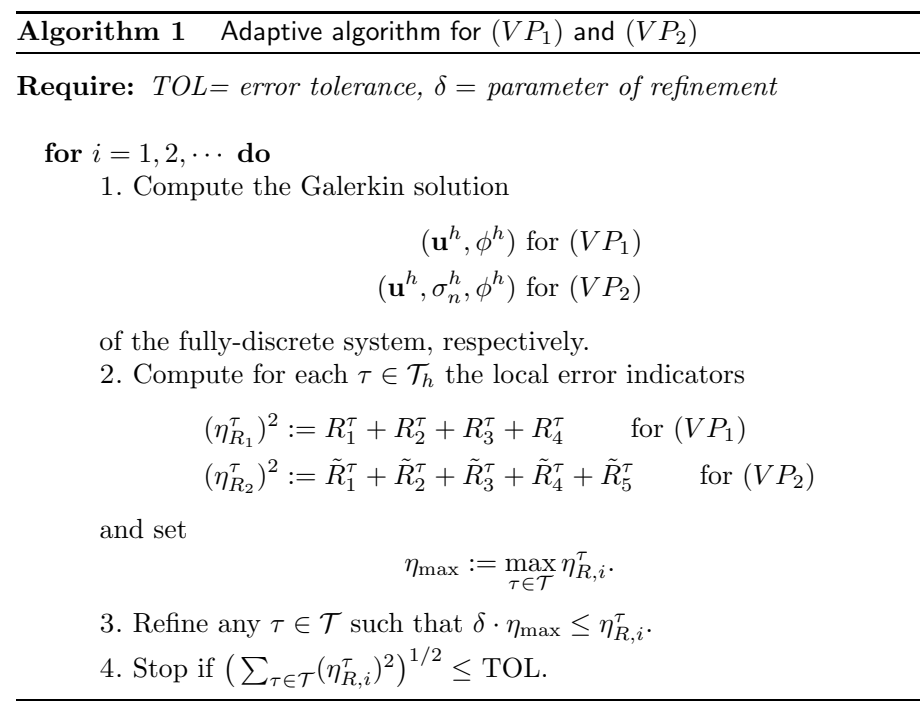


The implementation of this algorithm was performed using the program package MaiProgs [21]. For the two dimensional case we perform an adaptive algorithm using a blue-green refinement on triangles and for the three dimensional we allow hanging nodes on hexahedrons.

\section{Numerical Experiments}

Consider a square-shaped, homogeneous, isotropic, elastic scatterer made of steel with $\bar{\Omega}=$ $[-1,1]^{2}$ for the two dimensional case or $\bar{\Omega}=[-1,1]^{3}$ for the three dimensional case. The scatterer possesses the following material parameters: Poisson's ratio $\nu=0.28$, Young's module $E=200 G P a$ and $\rho=7800 \mathrm{~kg} / \mathrm{m}^{3}$. The scatterer is submerged in sea water and is subject to a plane incident wave $p^{0}\left(x_{1}, x_{2}\right)=e^{i k x_{1}}$ for the $2 \mathrm{D}$ case and $p^{0}\left(x_{1}, x_{2}, x_{3}\right)=e^{i k x_{1}}$ for the $3 \mathrm{D}$ case. Furthermore, we assume for sea water a density $\rho_{0}=1020 \mathrm{Kg} / \mathrm{m}^{3}$ and a sound velocity $c_{0}=1500 \mathrm{~m} / \mathrm{s}$. In the following let $\|\cdot\|_{W_{0}},\|\cdot\|_{1, W_{0}}$ and $\|\cdot\|_{1,1, W_{0}}$ denote norms defined by

$$
\begin{aligned}
\|\psi\|_{W_{0}}^{2} & :=\left\langle W_{0} \psi, \bar{\psi}\right\rangle \quad \forall \psi \in H^{1 / 2}(\Gamma), \\
\|(\mathbf{v}, \psi)\|_{1, W_{0}}^{2} & :=\|\mathbf{v}\|_{1}^{2}+\|\psi\|_{W_{0}}^{2} \quad \forall(\mathbf{v}, \psi) \in \mathcal{H}_{1}, \\
\|(\mathbf{v}, \chi, \psi)\|_{1, W_{0}, W_{0}}^{2} & =\|\mathbf{v}\|_{1}^{2}+\left\langle W_{0} \psi, \bar{\psi}\right\rangle+\left\langle W_{0} \chi, \bar{\chi}\right\rangle \quad \forall(\mathbf{v}, \chi, \psi) \in \mathcal{H}_{2},
\end{aligned}
$$

where $W_{0}$ is the hypersingular operator with kernel $\gamma_{k=0}$ (see eq. (7)). In order, to calculate the error estimators of the formulations $\left(V P_{1}\right)$ and $\left(V P_{2}\right)$ in the norm of $\mathcal{H}_{1}$ and $\mathcal{H}_{2}$ we use the equivalent norms $\|\cdot\|_{1, W_{0}}$ and $\|\cdot\|_{1, W_{0}, W_{0}}$, respectively.

Remark 5.1. We remark that for our numerical example we do not know the exact solution of the system. The error and convergence analysis for the numerical solutions is performed using estimates of the exact norms of $\mathbf{u} \in\left[H^{1}(\Omega)\right]^{2}, \sigma_{n} \in H^{1 / 2}(\Gamma)$ and $\phi \in H^{1 / 2}(\Gamma)$. These estimates are obtained by extrapolation using Aitken's $\Delta^{2}$ process with a sequence of norms, resulting from an h-uniformly refinement.

2D Case. Firstly, we compare the performance obtained with stable $(\operatorname{Im} \alpha \neq 0)$ and nonstable procedures. In Fig. 1 the convergence of error $e$ is shown for a $h$-uniform refinement in $\left(V P_{1}\right)$ and $\left(V P_{2}\right)$, respectively, using $\alpha=0$ and $\alpha=i / k$ and for the wave numbers $k=2,3.5$ and 5 . For the formulation $\left(V P_{1}\right)$ the error $e$ is given by $e:=\left\|(\mathbf{u}, \phi)-\left(\mathbf{u}^{h}, \phi^{h}\right)\right\|_{1, W_{0}}$ and for the formulation $\left(V P_{2}\right) e:=\left\|\left(\mathbf{u}, \sigma_{n}, \phi\right)-\left(\mathbf{u}^{h}, \sigma_{n}^{h}, \phi^{h}\right)\right\|_{1, W_{0}, W_{0}}$, respectively. As expected for $\alpha=0$ the method does not converge and for $\alpha=i / k$ the method converges. We choose the values $k$ close to a critical frequency of the system (see [22]).

Now we show the residual errors $\eta_{R_{1}}$ and $\eta_{R_{2}}$ as stated in Theorem 4.2 and Theorem 4.3 and apply the adaptive strategy in Algorithm 1. In Fig. 2 the error $e$ using an $h$-uniform and adaptive refinement, with their respective residual error estimator $\eta_{R_{1}}$ are displayed for different wave numbers $k$. Table I shows the residual error estimator $\eta_{R_{1}}$ and its effectivity index $\theta=\eta_{R_{1}} / e$ calculated for $k=3.5$ and $k=5$. We see that for $k=3.5$, the error $e$ has a slightly better convergence than the error estimator $\eta_{R_{1}}$. This difference may be explained by the lack of regularity of the solution $(\mathbf{u}, \phi)$ for this wave number. However for $k=5$ the equivalence between the error $e$ and $\eta_{R_{1}}$ is entirely confirmed. Note that in both cases Theorem 4.2 is confirmed. 

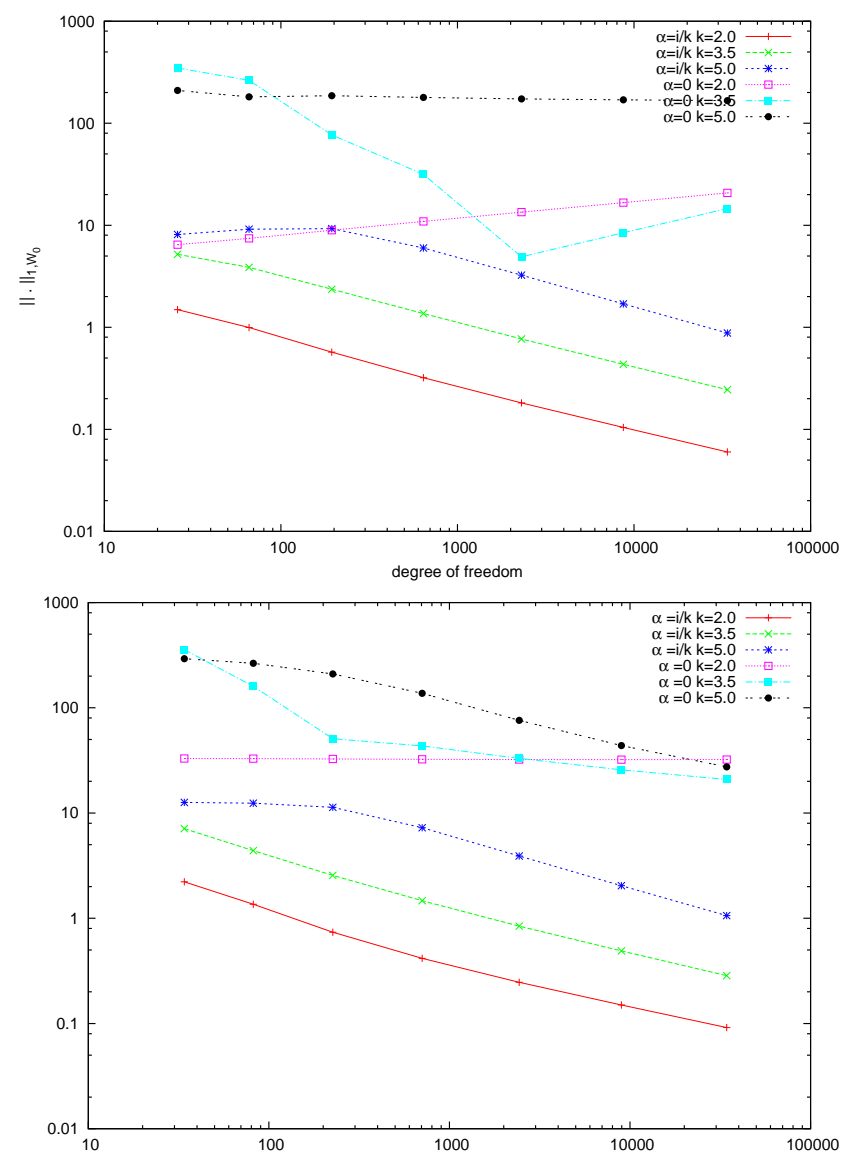

Figure 1. 2D: Error $e$ vs. degree of freedom using $\alpha=0$ and $\alpha=i / k$ (top: $\left(V P_{1}\right)$ case, bottom: $\begin{aligned}\left(V P_{2}\right) \text { case). With } \alpha=i / k: k=2-, k=5-\bullet, k=3.5-\times-, k=5-*-. & \text { With } \alpha=0: k=2-\square-, \\ k=3.5--, k & =5-\bullet .\end{aligned}$

Table I. 2D: Residual error estimator $\eta_{R_{1}}$ and effectivity index $\theta$ calculated for $k=3.5$ and $k=5$ with $\alpha=i / k$ using $\left(V P_{1}\right)$.

\begin{tabular}{cccccc}
\hline & \multicolumn{2}{c}{$k=3.5$} & \multicolumn{2}{c}{$k=5.0$} \\
\hline$h$ & $N$ & $\eta_{R_{1}}$ & $\theta=\eta_{R_{1}} / e$ & $\eta_{R_{1}}$ & $\theta=\eta_{R_{1}} / e$ \\
\hline 1 & 26 & 6.3622 & 1.2257 & 29.9753 & 3.6887 \\
$1 / 2$ & 66 & 5.5580 & 1.4366 & 31.5406 & 3.4438 \\
$1 / 4$ & 194 & 4.7024 & 1.9920 & 20.8456 & 2.2508 \\
$1 / 8$ & 642 & 3.2281 & 2.3674 & 13.0691 & 2.1793 \\
$1 / 16$ & 2306 & 1.9494 & 2.5368 & 7.1497 & 2.2010 \\
$1 / 32$ & 8706 & 1.1588 & 2.6715 & 3.7212 & 2.1949 \\
$1 / 64$ & 33794 & 0.7152 & 2.9234 & 1.9314 & 2.1949 \\
\hline
\end{tabular}



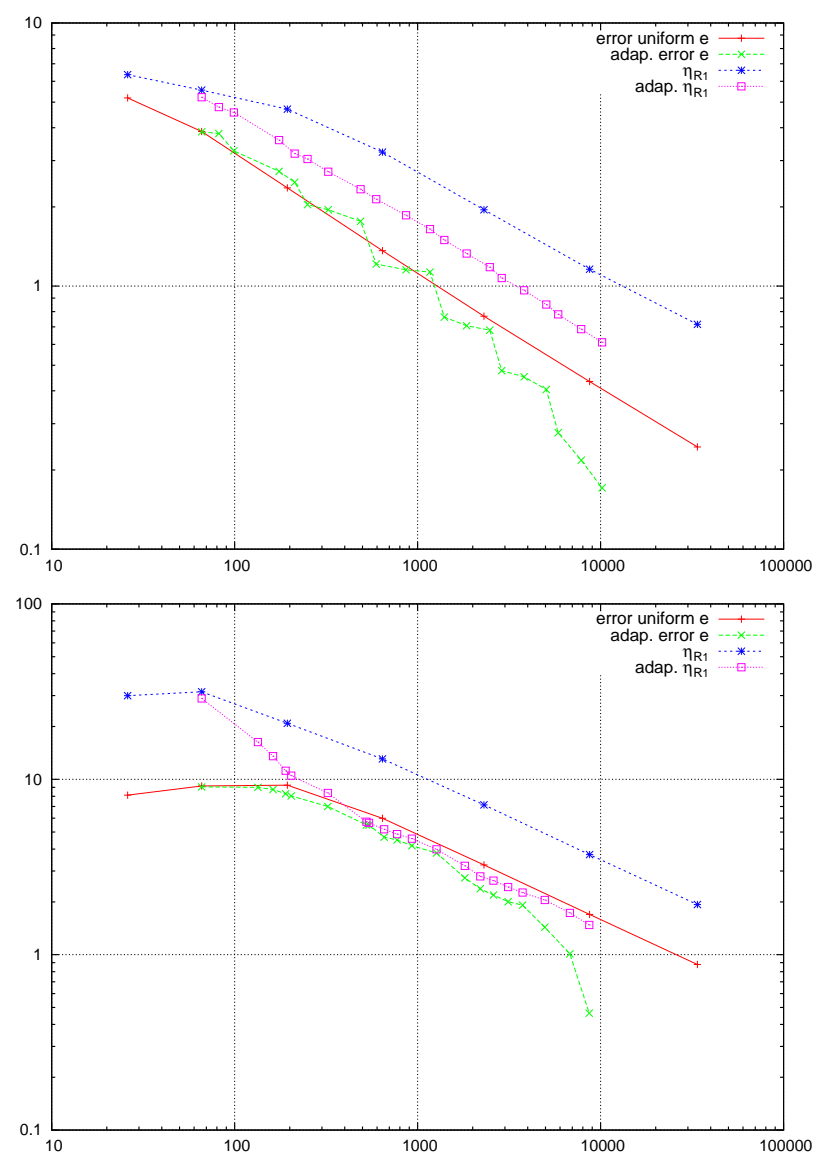

Figure 2. Errors and residual error estimators $\eta_{R_{1}}$ for the formulation $\left(V P_{1}\right)$ with $\alpha=i / k$ using $k=3.5$ (top) and $k=5.0$ (bottom). $-1-e$ using uniform refinement, $-\times e$ using adaptive refinement, $\rightarrow-\eta_{R_{1}}$ using uniform refinement, $-\square-\eta_{R_{1}}$ using adaptive refinement.

Fig. 3 shows the error $e:=\left\|\left(\mathbf{u}, \sigma_{n}, \phi\right)-\left(\mathbf{u}^{h}, \sigma_{n}^{h}, \phi^{h}\right)\right\|_{1, W_{0}, W_{0}}$, for the formulation $\left(V P_{2}\right)$ using a uniform and adaptive refinement, with their respective residual error estimator $\eta_{R_{2}}$ for different wave numbers $k$. Table II shows the residual error estimator $\eta_{R_{2}}$ and its effectivity index $\theta=\eta_{R_{2}} / e$ calculated for $k=3.5$ and $k=5$. We can see that the behavior is similar to the non-symmetric formulation $\left(V P_{1}\right)$. As in the formulation $\left(V P_{1}\right)$, for $k=3.5$ the error $e$ has a slightly better convergence than the error estimator $\eta_{R_{2}}$, and for $k=5$, the equivalence between the error $e$ and $\eta_{R_{2}}$ is entirely confirmed. Thus for this formulation, we can say that Theorem 4.3 is satisfied. Fig. 4 and 5 show different adaptive meshes for both procedures. Note the similarity of the areas of refinement for both procedures.

3D Case. For the three dimensional case we use hexahedral elements for the discretization of the domain $\Omega$ and squares for the discretization of the boundary $\Gamma$. The adaptive method uses hanging nodes for the construction of the mesh following the one-constraint rule, i.e., one 

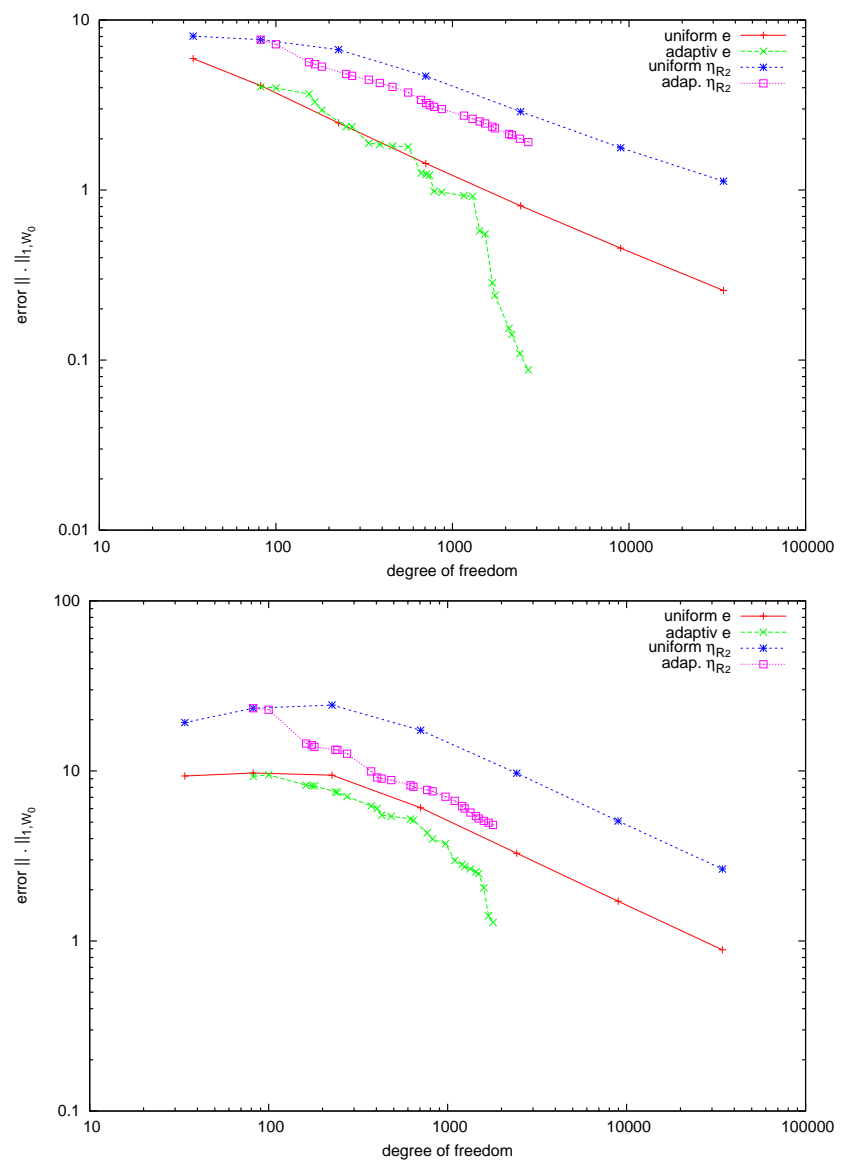

Figure 3. Errors and residual error estimators $\eta_{R_{2}}$ for the formulation $\left(V P_{2}\right)$ with $\alpha=i / k$ using $k=3.5$ (top) and $k=5$ (bottom). $-1-e$ using uniform refinement, $-\times-e$ using adaptive refinement, $-*-\eta_{R_{2}}$ using uniform refinement, $-\square-\eta_{R_{2}}$ using adaptive refinement

edge has at most two smaller neighboring edges on the other element. In Table III we show the residual error estimator obtained for $h$-uniform refinement. Here, we confirm the efficiency and reliability of our residual estimator $\eta_{R_{1}}$ obtained in $h$-uniform refinement. This confirms the efficiency of our residual estimator and the predicted order of convergence of our discrete problem. One can see that our estimate $\eta_{R_{1}}$ is proportional to $e$, since the effectivity index $\theta=\eta_{R_{1}} / e \approx 0.6$ shown in Table III is quasi-constant, so we verify Theorem 4.2 for the three dimensional version and the non-symmetric formulation $\left(V P_{1}\right)$.

Table IV shows the residual error estimator obtained for $h$-uniform refinement in the three dimensional case. Here, we confirm the efficiency of our residual estimator $\eta_{R_{2}}$ stated in Theorem 4.3 and the predicted order of convergence of the discrete solution. We can see that our estimate $\eta_{R_{2}}$ is proportional to $e$, since the effectivity index $q=\eta_{R_{2}} / e \approx 1.3$ shown in Table IV is quasi-constant. This verifies Theorem 4.3 for the three dimensional version and the symmetric formulation. Fig. 6 shows the error for $h$-uniform refinement using $\left(V P_{1}\right)$ and 
Table II. 2D: Residual error estimators and effectivity index $\theta$ using $\left(V P_{2}\right)$ with $\alpha=i / k, k=3.5$ and $k=5$.

\begin{tabular}{cccc}
\hline \multicolumn{2}{c}{$k=3.5$} & \multicolumn{2}{c}{$k=5$} \\
\hline$\eta_{R_{2}}$ & $\theta=\eta_{R_{2}} / e$ & $\eta_{R_{2}}$ & $\theta=\eta_{R_{2}} / e$ \\
\hline 1.3556 & 1.3556 & 19.2485 & 2.0610 \\
1.8644 & 1.8644 & 23.3774 & 2.4047 \\
2.6932 & 2.6932 & 24.4007 & 2.5826 \\
3.2610 & 3.2610 & 17.3447 & 2.8555 \\
2.8919 & 3.5822 & 9.6990 & 2.9545 \\
1.7716 & 3.8935 & 5.0793 & 2.9670 \\
1.1263 & 4.3944 & 2.6479 & 2.9846 \\
\hline
\end{tabular}

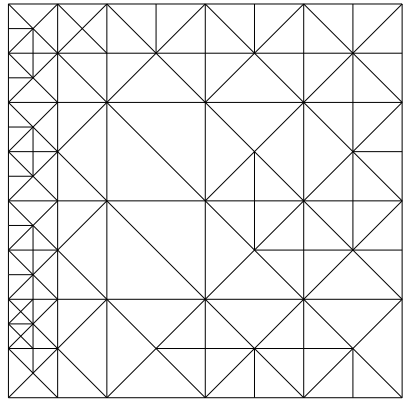

$N=213$ dof

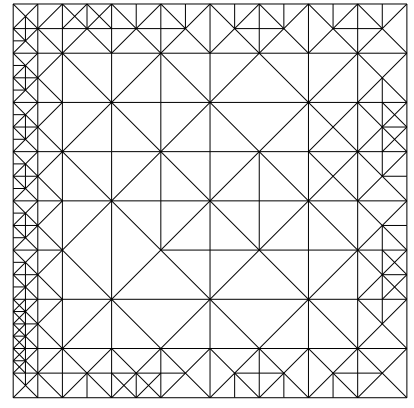

$N=488$ dof

Figure 4. 2D: Adaptive meshes using $\left(V P_{1}\right)$ with $k=3.5$ and parameter of refinement $\delta=0.8$.

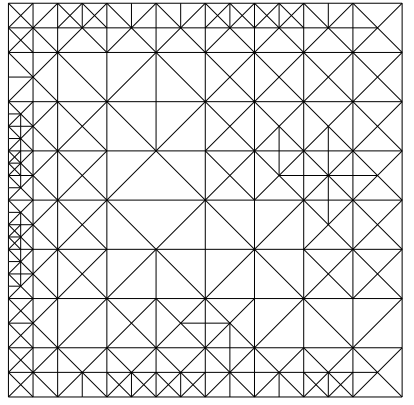

$N=562$ dof

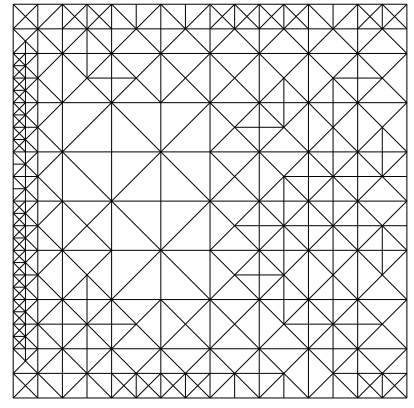

$N=1470$ dof

Figure 5. 2D: Adaptive meshes using $\left(V P_{2}\right)$ with $k=3.5$ and parameter of refinement $\delta=0.9$. 
Table III. 3D: Residual error estimator $\eta_{R_{1}}$ and effectivity index $\theta$ calculated for $k=5.2$ with $\alpha=i / k$ using $\left(V P_{1}\right)$.

\begin{tabular}{cccc}
\hline$h$ & $N$ & $\eta_{R_{1}}$ & $\theta=\eta_{R_{1}} / e$ \\
\hline 1 & 107 & 78.6972 & 4.4117 \\
$1 / 2$ & 473 & 28.6879 & 1.2164 \\
$1 / 3$ & 1247 & 17.0375 & 0.8621 \\
$1 / 4$ & 2573 & 11.8714 & 0.7431 \\
$1 / 5$ & 4595 & 9.2147 & 0.6955 \\
$1 / 6$ & 7457 & 7.5367 & 0.6729 \\
$1 / 7$ & 11303 & 6.3806 & 0.6646 \\
$1 / 8$ & 16277 & 5.5392 & 0.6666 \\
$1 / 9$ & 22523 & 4.9021 & 0.6775 \\
$1 / 10$ & 30185 & 4.4047 & 0.6972 \\
$1 / 11$ & 39407 & 4.0066 & 0.7267 \\
\hline
\end{tabular}

Table IV. 3D: Residual error estimator $\eta_{R_{2}}$ and effectivity index $\theta$ calculated using $\left(V P_{2}\right)$ for $k=5.2$ with $\alpha=i / k$.

\begin{tabular}{cccc}
\hline$h$ & $N$ & $\eta_{R_{2}}$ & $\theta=\eta_{R_{2}} / e$ \\
\hline 1 & 133 & 126.5869 & 5.4076 \\
$1 / 2$ & 571 & 52.9663 & 2.1531 \\
$1 / 3$ & 1465 & 32.7106 & 1.6537 \\
$1 / 4$ & 2959 & 23.3245 & 1.4628 \\
$1 / 5$ & 5197 & 18.1849 & 1.3803 \\
$1 / 6$ & 8323 & 14.8992 & 1.3442 \\
$1 / 7$ & 12481 & 12.6254 & 1.3371 \\
$1 / 8$ & 17815 & 10.9673 & 1.3529 \\
$1 / 9$ & 24469 & 9.7105 & 1.3903 \\
$1 / 10$ & 32587 & 8.7284 & 1.4517 \\
\hline
\end{tabular}

$\left(V P_{2}\right)$ with their respective residual error estimators $\eta_{R_{1}}$ and $\eta_{R_{2}}$.

Fig. 7 shows the error using $h$-uniform refinement and adaptive refinement with the L-Block $\Omega:=[-1,1] \backslash([0,1] \times[-1,1])$. After several refinements the error of the adaptive algorithm is less than the error in the uniform refinement. Note, that for adaptive meshes the residual error estimator is larger than the estimator obtained for uniform meshes. This appears to be caused by contribution of hanging nodes. Nonetheless the error for the adaptive scheme is smaller than for the uniform $h$-version. Our adaptive algorithm produces a sequence of refined meshes, which is shown in Fig. 8. 


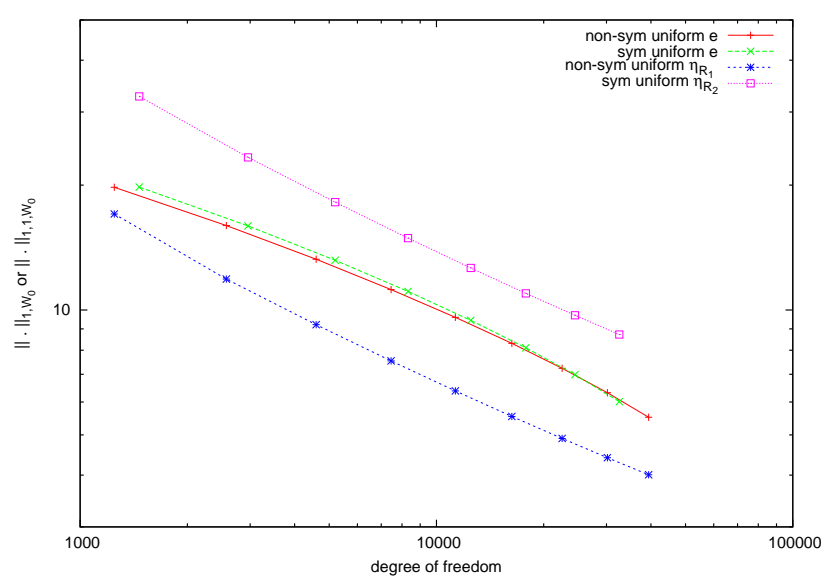

Figure 6. 3D: Error $e$ and error residual estimators of uniform refinements in $[-1,1]^{3}$ with $k=5.2$ and $\alpha=i / k$. - + $e$ using $\left(V P_{1}\right),-x-e$ using $\left(V P_{2}\right),-*-\eta_{R_{1}}$ using $\left(V P_{1}\right),-\square-\eta_{R_{2}}$ using $\left(V P_{2}\right)$.

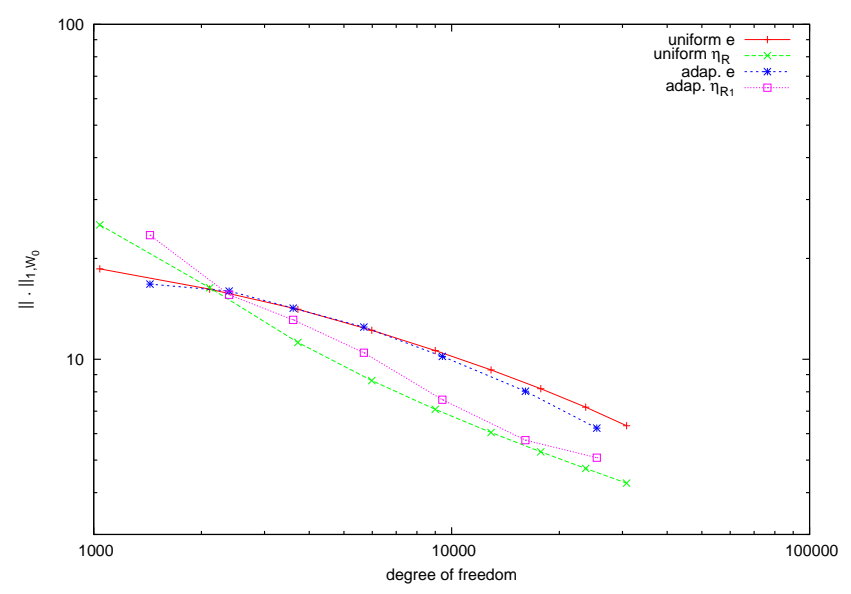

Figure 7. 3D: Error $e$ and error residual estimators $\eta_{R_{1}}$ of uniform and adaptive refinements in the L-Block using $\left(V P_{1}\right)$ with $k=5.2 \alpha=i / k$ and parameter of refinement $\delta=0.9 .-+e$ using uniform ref., $-\times-\eta_{R_{1}}$ using uniform ref., $-*-e$ using adaptive ref., $-\square-\eta_{R_{1}}$ using adaptive ref.

\section{Conclusion}

This paper develops reliable residual-based a posteriori error estimates for a finite element/boundary element formulation of the Lamé-Helmholtz coupled problem in 2D and 3D. This model represents the time-harmonic fluid-solid interaction between an inviscid unbounded exterior acoustic medium and a bounded elastic solid. We are using two different approaches to ensure uniqueness of the boundary integral equation part, which have been discussed in the literature before $([4,2,1])$, but our work is the first to analyze the residual error estimator in $2 \mathrm{D}$ and $3 \mathrm{D}$. 


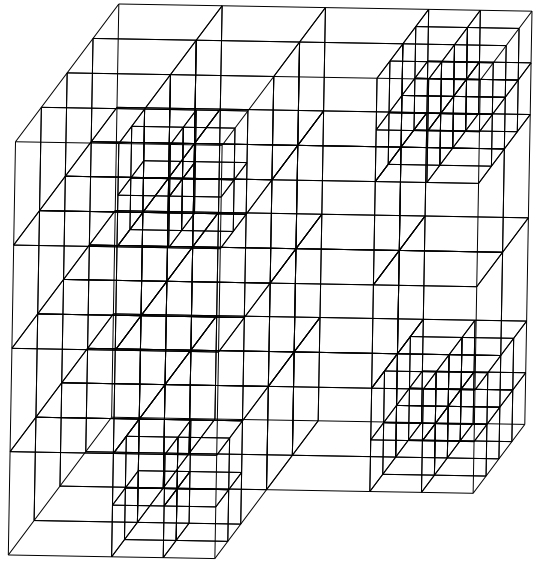

$N=589$ dof

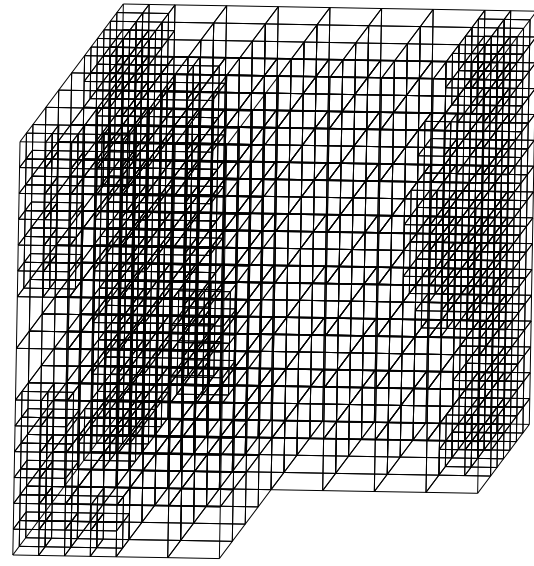

$N=3609$ dof

Figure 8. 3D: Sequence of meshes for the adaptive strategy using $\left(V P_{1}\right)$ with $k=5.2, \alpha=i / k$ and parameter of refinement $\delta=0.9$ on the L-Block.

This work contributes to the development of new numerical schemes for fluid-structure interactions, in particular, schemes involving the Helmholtz equation. Such schemes are widely known among engineers and mathematicians to be difficult to treat by standard numerical methods, due to non-uniqueness caused by the occurrence of resonance frequencies. The results presented here are a basis for further research on numerical models that involve non-viscous fluids and/or non-linear elastic solids using finite elements and/or boundary elements.

\section{REFERENCES}

1. Bielak J, MacCamy RC. Symmetric finite element and boundary integral coupling methods for fluid-solid interaction. Quart. Appl. Math. 1991; XLIX(1):107-119.

2. Bielak J, MacCamy RC, Zeng X. Stable coupling method for the interface scattering problems by combined integral equations and finite elements. Journal of Computational Physics 1995; 119:374-384.

3. Zeng X, Bielak J, MacCamy RC. Unified symmetric finite element and boundary integral variational coupling methods for linear fluid-structure interaction. Numer. Methods Partial Differential Equations 1992; 8(5):451-467.

4. Hsiao G, Kleinman R, Roach G. Weak solutions of fluid-solid interaction problems. Math. Nachr. 2000; 218(1):139-163

5. Burton AJ, Miller GF. The application of integral equation methods to the numerical solution of some exterior Boundary-Value problems. Proceedings of the Royal Society of London. Series A, Mathematical and Physical Sciences 1971; 323(1553):201-210.

6. Brakhage $\mathrm{H}$, Werner $\mathrm{P}$. über das dirichletsche außenraumproblem für die helmholtzsche schwingungsgleichung. Archiv der Mathematik 1965; 16(1):325-329.

7. Kress R. Minimizing the condition number of boundary integral operators in acoustic and electromagnetic scattering. Q J Mechanics Appl Math May 1985;38(2):323-341.

8. Chang YC, Demkowicz L. Solution of viscoelastic scattering problems in linear acoustics using $h p$ boundary/finite element method. International Journal for Numerical Methods in Engineering 1999; 44(12):1885-1907.

9. Gatica GN, Márquez A, Meddahi S. Analysis of the coupling of primal and dual-mixed finite element methods for a two-dimensional fluid-solid interaction problem. SIAM J. Numer. Anal. 2007; 45(5):20722097. 
10. Johnson C, Hansbo P. Adaptive finite element methods in computational mechanics. Comput. Methods Appl. Mech. Engrg. 1992; 101(1-3):143-181.

11. Stewart JR, Hughes TJR. An a posteriori error estimator and $h p$-adaptive strategy for finite element discretizations of the Helmholtz equation in exterior domains. Adaptive meshing, Part 1. Finite Elem. Anal. Des. 1997; 25(1-2):1-26.

12. Carstensen C, Stephan EP. Adaptive boundary element methods for some first kind integral equations. SIAM Journal on Numerical Analysis 1996; 33(6):2166.

13. Carstensen C, Stephan EP. Adaptive coupling of boundary elements and finite elements. RAIRO Modél. Math. Anal. Numér. 1995; 29(7):779-817.

14. Carstensen C, Stephan EP. A posteriori error estimates for boundary element methods. Mathematics of Computation 1995; 64(210):483-500.

15. Costabel M, Stephan EP. Duality estimates for the numerical solution of integral equations. Numerische Mathematik May 1989; 54(3):339-353.

16. Luke CJ, Martin PA. Fluid-solid interaction: acoustic scattering by a smooth elastic obstacle. SIAM J. Appl. Math. 1995; 55(4):904-922.

17. Colton D, Kress R. Inverse Acoustic and Electromagnetic Scattering Theory (2nd edition). Springer, 1998.

18. Hsiao GC, Wendland WL. Boundary integral equations. Springer, 2008.

19. MacCamy RC, Stephan EP. A boundary element method for an exterior problem for three-dimensional Maxwell's equations. Applicable Anal. 1983; 16(2):141-163.

20. Carstensen C, Funken SA, Stephan EP. On the adaptive coupling of FEM and BEM in 2-d-elasticity. Numer. Math. 1997; 77(2):187-221.

21. Maischak M. Technical manual of the program system MaiProgs. Technical report, Institut für Angewandte Mathematik, Universität Hannover, 2003.

22. C. Domínguez. Finite element and boundary element coupling for fluid-structure interaction. Phd thesis, Leibniz Hannover Universität, 2010.

23. Verfürth R. A Review of A Posteriori Error Estimation and Adaptive Mesh- Refinement Techniques. Teubner Verlag, 1996.

24. Carstensen C. A posteriori error estimate for the symmetric coupling of finite elements and boundary elements. Computing Dec. 1996; 57(4):301-322.

25. Hsiao GC, Wendland WL. The Aubin-Nitsche lemma for integral equations. J. Integral Equations 1981; $\mathbf{3}(4): 299-315$.

\section{APPENDIX}

Below we prove the efficiency of the residual error estimator $\eta_{R_{1}}$ for the formulation $\left(V P_{1}\right)$ on quasi-uniform meshes. The ideas of this proof can be found in Verfürth [23] for the indicators of the FEM part. For the indicators with boundary integral operators, we use some ideas of Carstensen [24]. For more detail see Domínguez [22]. Initially, we present a local upper bound for the error indicators $R_{1}^{h}$ and $R_{2}^{h}$ (see (14)).

Lemma I.1. Let $\tau \in \mathcal{T}_{h}$ and $R_{1, \tau}^{h}:=h_{\tau}^{2}\left\|\operatorname{div} \sigma\left(\mathbf{u}^{h}\right)+\rho \omega^{2} \mathbf{u}^{h}-\mathbf{f}\right\|_{0, \tau}^{2}$, then there holds

$$
R_{1, \tau}^{h} \lesssim\left\|\sigma\left(\mathbf{u}^{h}-\mathbf{u}\right)\right\|_{0, \tau}^{2}+h_{\tau}^{2}\left\|\mathbf{u}^{h}-\mathbf{u}\right\|_{0, \tau}^{2} .
$$

Proof. For the case $d=2$, we denote by $\lambda_{\tau, 1}, \lambda_{\tau, 2}, \lambda_{\tau, 3}$ the barycentric coordinates of the triangle $\tau \in \mathcal{T}_{h}$. We define the triangle-bubble function $b_{\tau}$ by

$$
b_{\tau}:= \begin{cases}27 \lambda_{\tau, 1} \lambda_{\tau, 2} \lambda_{\tau, 3} & \text { on } \tau \\ 0 & \text { on } \Omega \backslash \tau .\end{cases}
$$

For $d=3$ we denote by $\lambda_{\tau, 1}, \lambda_{\tau, 2}, \lambda_{\tau, 3}, \lambda_{\tau, 4}$ the barycentric coordinates of a tetrahedron $\tau \in \mathcal{T}_{h}$. We define the bubble function $b_{\tau}$ by

$$
b_{\tau}:= \begin{cases}256 \lambda_{\tau, 1} \lambda_{\tau, 2} \lambda_{\tau, 3} \lambda_{\tau, 4} & \text { on } \tau \\ 0 & \text { on } \Omega \backslash \tau .\end{cases}
$$


In both cases $(d=2,3)$ the function $b_{\tau}$ has the following properties (see Verfürth [23, p. 10]) with a constant $c>0$.

$$
\begin{gathered}
\operatorname{supp} b_{\tau} \subset \tau, \quad 0 \leq b_{\tau} \leq 1, \quad \max _{x \in \tau} b_{\tau}(x)=1, \\
\left\|b_{\tau}^{1 / 2} \mathbf{u}\right\|_{0, \tau} \leq\|\mathbf{u}\|_{0, \tau} \leq c\left\|b_{\tau}^{1 / 2} \mathbf{u}\right\|_{0, \tau}, \quad\left\|\nabla b_{\tau}^{1 / 2} \mathbf{u}\right\|_{0, \tau} \leq h_{\tau}^{-1}\|\mathbf{u}\|_{0, \tau} .
\end{gathered}
$$

We define $g_{\tau}:=\left(\operatorname{div} \sigma\left(\mathbf{u}^{h}\right)+\rho \omega^{2} \mathbf{u}^{h}-\mathbf{f}\right) b_{\tau}$. Then from $(24)$

$$
\begin{aligned}
\| \operatorname{div} \sigma\left(\mathbf{u}^{h}\right) & +\rho \omega^{2} \mathbf{u}^{h}-\mathbf{f} \|_{0, \tau}^{2} \\
& \lesssim\left\|\left(\operatorname{div} \sigma\left(\mathbf{u}^{h}\right)+\rho \omega^{2} \mathbf{u}^{h}-\mathbf{f}\right) b_{\tau}^{1 / 2}\right\|_{0, \tau}^{2} \\
& =\int_{\tau}\left(\operatorname{div} \sigma\left(\mathbf{u}^{h}\right)+\rho \omega^{2} \mathbf{u}^{h}-\mathbf{f}\right) g_{\tau} d x \\
& =\int_{\tau}\left(\operatorname{div} \sigma\left(\mathbf{u}^{h}\right)+\rho \omega^{2} \mathbf{u}^{h}\right) g_{\tau} d x-\int_{\tau}\left(\operatorname{div} \sigma(\mathbf{u})+\rho \omega^{2} \mathbf{u}\right) g_{\tau} d x \\
& =\int_{\tau}\left(\sigma\left(\mathbf{u}-\mathbf{u}^{h}\right): \nabla g_{\tau}+\rho \omega^{2}\left(\mathbf{u}^{h}-\mathbf{u}\right) g_{\tau}\right) d x \\
& \lesssim\left\|\sigma\left(\mathbf{u}^{h}-\mathbf{u}\right)\right\|_{0, \tau}\left\|\nabla g_{\tau}\right\|_{0, \tau}+\left\|\mathbf{u}^{h}-\mathbf{u}\right\|_{0, \tau}\left\|g_{\tau}\right\|_{0, \tau} \\
& \lesssim\left\|g_{\tau}\right\|_{0, \tau}\left(h_{\tau}^{-1}\left\|\sigma\left(\mathbf{u}^{h}-\mathbf{u}\right)\right\|_{0, \tau}+\left\|\mathbf{u}^{h}-\mathbf{u}\right\|_{0, \tau}\right) \\
& \lesssim\left\|\operatorname{div} \sigma\left(\mathbf{u}^{h}\right)+\rho \omega^{2} \mathbf{u}^{h}-\mathbf{f}\right\|_{0, \tau}\left(h_{\tau}^{-1}\left\|\sigma\left(\mathbf{u}^{h}-\mathbf{u}\right)\right\|_{0, \tau}+\left\|\mathbf{u}^{h}-\mathbf{u}\right\|_{0, \tau}\right) .
\end{aligned}
$$

Dividing the last inequality by $\left\|\operatorname{div} \sigma\left(\mathbf{u}^{h}\right)+\rho \omega^{2} \mathbf{u}^{h}-\mathbf{f}\right\|_{0, \tau}$ it follows that

$$
\left\|\operatorname{div} \sigma\left(\mathbf{u}^{h}\right)+\rho \omega^{2} \mathbf{u}^{h}-\mathbf{f}\right\|_{0, \tau} \leq c\left(h_{\tau}^{-1}\left\|\sigma\left(\mathbf{u}^{h}-\mathbf{u}\right)\right\|_{0, \tau}+\left\|\mathbf{u}^{h}-\mathbf{u}\right\|_{0, \tau}\right) .
$$

Finally, raising powers to the square and multiplying by $h_{\tau}^{2}$ we obtain $(21)$.

Next, we estimate the local indicator $R_{2, \tau}^{h}$ related to the jump on $s \in \mathcal{S}_{i, \tau}$.

Lemma I.2. Let $\tau \in \mathcal{T}_{h}$ then there holds

$$
R_{2, \tau}^{h}:=\sum_{s_{i} \in \tau} h_{s}\left\|\llbracket \sigma\left(\mathbf{u}^{h}\right) \mathbf{n} \rrbracket\right\|_{0, s}^{2} \lesssim\left\|\sigma\left(\mathbf{u}-\mathbf{u}^{h}\right)\right\|_{0, w_{\tau}}^{2}+h_{s}^{2}\|\mathbf{u}\|_{0, w_{\tau}}^{2},
$$

where $w_{\tau}$ are the element neighbors of $\tau$.

Proof. We estimate the indicator $R_{2}^{h}$ related to the jump in $s \in \mathcal{S}_{i}$ with $s=\partial \tau_{1} \cap \partial \tau_{2}$, where $\tau_{1}$ and $\tau_{2}$ are the elements that contain the face $s$. Let $w_{s}:=\tau_{1} \cup \tau_{2}$. For this we need the following definitions: Considering the two-dimensional case, we define an edge-bubble function $b_{s}$ (see Verfürth [23][p. 10]) by

$$
b_{s}:= \begin{cases}4 \lambda_{\tau_{i, 1}} \lambda_{\tau_{i, 2}} & \text { on } \tau_{i}, \quad i=1,2, \\ 0 & \text { on } \Omega \backslash w_{s},\end{cases}
$$

where $\lambda_{\tau_{i, 1}}, \lambda_{\tau_{i, 2}}$ are the barycentric coordinates of $\tau_{i}(i=1,2)$ related to the edge $s$. For the three-dimensional case, we define a face-bubble function $b_{s}$ with barycentric coordinates $\lambda_{\tau_{i, 1}}, \lambda_{\tau_{i, 2}}, \lambda_{\tau_{i, 3}}$

$$
b_{s}:= \begin{cases}27 \lambda_{\tau_{i, 1}} \lambda_{\tau_{i, 2}} \lambda_{\tau_{i, 3}} & \text { on } s \in \mathcal{S}_{i} \\ 0 & \text { on } \Omega \backslash s .\end{cases}
$$


In the case $d=2$ the function $b_{s}$ has the following properties:

$$
\begin{aligned}
& \operatorname{supp} b_{s} \subset w_{s}, \quad 0 \leq b_{s} \leq 1, \quad \max _{x \in s} b_{s}(x)=1, \quad \int_{s} b_{s}=\frac{2}{3} h_{s}, \\
& c_{1} h_{s}^{2} \leq \int_{\tau} b_{s}=\frac{1}{3}|\tau| \leq c_{2} h_{s}^{2}, \quad\left\|\nabla b_{s}\right\|_{0, \tau} \leq c_{3} h_{s}^{-1}\left\|b_{s}\right\|_{0, \tau} \quad \forall \tau \in w_{s} .
\end{aligned}
$$

For the case $d=3, b_{s}$ is the same triangle-bubble function defined in $(22)$, therefore $b_{s}$ satisfies the properties (24).

We have to prove the following result:

$$
\left\|\llbracket \sigma\left(\mathbf{u}^{h}\right) \mathbf{n} \rrbracket\right\|_{0, s}^{2} \lesssim h_{s}^{-1}\left\|\sigma\left(\mathbf{u}-\mathbf{u}^{h}\right)\right\|_{0, w_{s}}^{2}+h_{s}\|\mathbf{u}\|_{0, w_{s}}^{2} .
$$

We define $g_{s}:=\llbracket \sigma\left(\mathbf{u}^{h}\right) \mathbf{n} \rrbracket b_{s}$. Since we use continuous linear functions, the term $\llbracket \sigma\left(\mathbf{u}^{h}\right) \cdot \mathbf{n} \rrbracket$ is a complex number, then (26) implies that

$$
\int_{s} \llbracket \sigma\left(\mathbf{u}^{h}\right) \mathbf{n} \rrbracket g_{s}=\frac{2}{3} h_{s}\left\|\left.\llbracket \sigma\left(\mathbf{u}^{h}\right) \mathbf{n} \rrbracket\right|^{2}=\frac{2}{3}\right\| \llbracket \sigma\left(\mathbf{u}^{h}\right) \mathbf{n} \rrbracket \|_{0, s}^{2} .
$$

Using Green's Theorem, (3a), the Cauchy-Schwarz inequality, properties (26), (28) and taking in account that $\operatorname{div} \sigma\left(\mathbf{u}^{h}\right)=\mathbf{0}$ we obtain

$$
\begin{aligned}
\left\|\llbracket \sigma\left(\mathbf{u}^{h}\right) \mathbf{n} \rrbracket b_{s}^{1 / 2}\right\|_{0, s}^{2}= & \int_{w_{s}} \sigma\left(\mathbf{u}^{h}\right): \nabla g_{s} d x+\int_{w_{s}} \operatorname{div} \sigma\left(\mathbf{u}^{h}\right) \cdot g_{s} d x-\int_{\partial w_{s}} \sigma\left(\mathbf{u}^{h}\right) \mathbf{n} \cdot g_{s} \\
= & \int_{w_{s}} \sigma\left(\mathbf{u}^{h}\right): \nabla g_{s} d x \\
& -\int_{w_{s}} \sigma(\mathbf{u}): \nabla g_{s} d x-\int_{w_{s}} \operatorname{div} \sigma(\mathbf{u}) \cdot g_{s} d x+\int_{\partial w_{s}} \sigma(\mathbf{u}) \mathbf{n} \cdot g_{s} \\
= & \int_{w_{s}} \sigma\left(\mathbf{u}^{h}-\mathbf{u}\right): \nabla g_{s} d x+\rho \omega^{2} \int_{w_{s}} \mathbf{u} \cdot g_{s} d x \\
\lesssim & \left\|\sigma\left(\mathbf{u}^{h}-\mathbf{u}\right)\right\|_{0, w_{s}}\left\|\nabla g_{s}\right\|_{0, w_{s}}+\|\mathbf{u}\|_{0, w_{s}}\left\|g_{s}\right\|_{0, w_{s}} \\
\lesssim & h_{s}^{-1}\left|\llbracket \sigma\left(\mathbf{u}^{h}\right) \mathbf{n} \rrbracket\right|\left\|b_{s}\right\|_{0, s}\left\|\sigma\left(\mathbf{u}^{h}-\mathbf{u}\right)\right\|_{0, w_{s}} \\
& +\left|\llbracket \sigma\left(\mathbf{u}^{h}\right) \mathbf{n} \rrbracket\right|\left\|b_{s}\right\|_{0, s}\|\mathbf{u}\|_{0, w_{s}} \\
\lesssim & \left\|\llbracket \sigma\left(\mathbf{u}^{h}\right) \mathbf{n} \rrbracket\right\|_{0, s}\left(\int_{w_{s}} b_{s}\right)^{1 / 2}\left(h_{s}^{-3 / 2}\left\|\sigma\left(\mathbf{u}^{h}-\mathbf{u}\right)\right\|_{0, w_{s}}+h_{s}^{-1 / 2}\|\mathbf{u}\|_{0, w_{s}}\right) \\
\lesssim & \left\|\llbracket \sigma\left(\mathbf{u}^{h}\right) \mathbf{n} \rrbracket\right\|_{0, s}\left(h_{s}^{-1 / 2}\left\|\sigma\left(\mathbf{u}^{h}-\mathbf{u}\right)\right\|_{0, w_{s}}+h_{s}^{1 / 2}\|\mathbf{u}\|_{0, w_{s}}\right)
\end{aligned}
$$

Dividing the last inequality by $\left\|\llbracket \sigma\left(\mathbf{u}^{h}\right) \cdot \mathbf{n} \rrbracket\right\|_{0, s}$, raising powers to the square and multiplying by $h_{s}$ yields (27). Finally, summing over each interior edge of $\tau$ result (25) follows.

Summing the local estimators from Lemma I.1 and I.2 we obtain the following result. Since $\|\cdot\|_{0, \Omega}^{2}=\sum_{\tau \in \mathcal{T}_{h}}\|\cdot\|_{0, \tau}^{2}$ it follows

Lemma I.3. There holds

$$
\begin{aligned}
& R_{1}^{h} \lesssim\left\|\sigma\left(\mathbf{u}^{h}-\mathbf{u}\right)\right\|_{0, \Omega}^{2}+h_{\max , \Omega}^{2}\left\|\mathbf{u}^{h}-\mathbf{u}\right\|_{0, \Omega}^{2}, \\
& R_{2}^{h} \lesssim\left\|\sigma\left(\mathbf{u}-\mathbf{u}^{h}\right)\right\|_{0, \Omega}^{2}+h_{\max , \Omega}^{2}\|\mathbf{u}\|_{0, \Omega}^{2},
\end{aligned}
$$

where $h_{\max , \Omega}$ is the maximum length of the regular decomposition $\mathcal{T}_{h}$ of $\Omega$. 
Next, we give upper local estimates for the estimators $R_{3}^{h}$ and $R_{4}^{h}$. Since we use $(2, r)$-regular boundary element $\mathcal{B}^{h}$ families in the sense of Babuška and Aziz, one can assume the inverse assumption. For more details see e.g. Hsiao and Wendland [25].

Lemma I.4. Inverse assumption: For $m \leq s \leq 2,|m|,|s| \leq r$ there exists a constant $c=c(m, s, r)$ for all $\eta^{h} \in \mathcal{B}^{h}$

$$
\left\|\eta^{h}\right\|_{s} \leq c h^{t-s}\left\|\eta^{h}\right\|_{t} \quad \forall \eta \in \mathcal{B}^{h} .
$$

Lemma I.5. Assuming $(\mathbf{u}, \phi) \in\left[H^{2}(\Omega)\right]^{d} \times H^{3 / 2}(\Gamma)$ it follows

$$
R_{3}^{h} \lesssim h_{\max , \Gamma}\left(\left\|\sigma\left(\mathbf{u}-\mathbf{u}^{h}\right)\right\|_{0, \Gamma}^{2}+\left\|\phi-\phi^{h}\right\|_{0, \Gamma}^{2}+\left\|\phi-\phi^{h}\right\|_{-1, \Gamma}^{2}\right),
$$

where $h_{\max , \Gamma}$ is the maximum length of the regular decomposition $\mathcal{S}_{\Gamma, \tilde{h}}$ of $\Gamma$.

Proof. Noting that $p^{0} \mathbf{n}=-\sigma(\mathbf{u}) \mathbf{n}+\left(V+\alpha\left(K+\frac{I}{2}\right)\right)(\phi) \mathbf{n}$, we obtain in each $s \in \mathcal{S}_{\Gamma, \tilde{h}}$

$$
\begin{aligned}
\| \sigma\left(\mathbf{u}^{h}\right) \mathbf{n} & -p^{0} \mathbf{n}-V \phi^{h} \mathbf{n}-\alpha\left(K+\frac{I}{2}\right) \phi^{h} \mathbf{n} \|_{0, s}^{2} \\
& \lesssim\left\|\sigma\left(\mathbf{u}-\mathbf{u}^{h}\right) \mathbf{n}\right\|_{0, s}^{2}+\left\|V\left(\phi-\phi^{h}\right) \mathbf{n}\right\|_{0, s}^{2}+\left\|\alpha\left(K+\frac{I}{2}\right)\left(\phi-\phi^{h}\right) \mathbf{n}\right\|_{0, s}^{2} .
\end{aligned}
$$

Summing the estimate (30) over all elements $s \in \mathcal{S}_{\Gamma, \tilde{h}}$ and due to $\|\cdot\|_{0, \Gamma}^{2}=\sum_{s \in \mathcal{S}_{\Gamma, \tilde{h}}}\|\cdot\|_{0, s}^{2}$ we obtain

$$
R_{3}^{h} \lesssim h_{\max , \Gamma}\left(\left\|\sigma\left(\mathbf{u}-\mathbf{u}^{h}\right) \mathbf{n}\right\|_{0, \Gamma}^{2}+\left\|V\left(\phi-\phi^{h}\right) \mathbf{n}\right\|_{0, \Gamma}^{2}+\left\|\alpha\left(K+\frac{I}{2}\right)\left(\phi-\phi^{h}\right) \mathbf{n}\right\|_{0, \Gamma}^{2}\right) .
$$

Since $V: H^{-1 / 2+\tilde{s}}(\Gamma) \rightarrow H^{1 / 2+\tilde{s}}(\Gamma)$ and $K: H^{1 / 2+\tilde{s}}(\Gamma) \rightarrow H^{1 / 2+\tilde{s}}(\Gamma)$ are continuous mappings for $\tilde{s} \in[-1 / 2,1 / 2]$ we have that

$$
\begin{gathered}
\left\|V\left(\phi-\phi^{h}\right) \mathbf{n}\right\|_{0, \Gamma}^{2} \lesssim\left\|V\left(\phi-\phi^{h}\right)\right\|_{0, \Gamma}^{2} \lesssim\left\|\phi-\phi^{h}\right\|_{-1, \Gamma}^{2}, \\
\left\|\left(K+\frac{I}{2}\right)\left(\phi-\phi^{h}\right) \mathbf{n}\right\|_{0, \Gamma}^{2} \lesssim\left\|\left(K+\frac{I}{2}\right)\left(\phi-\phi^{h}\right)\right\|_{0, \Gamma}^{2} \lesssim\left\|\phi-\phi^{h}\right\|_{0, \Gamma}^{2} .
\end{gathered}
$$

Thus

$$
R_{3}^{h} \lesssim h_{\max , \Gamma}\left(\left\|\sigma\left(\mathbf{u}-\mathbf{u}^{h}\right)\right\|_{0, \Gamma}^{2}+\left\|\phi-\phi^{h}\right\|_{0, \Gamma}^{2}+\left\|\phi-\phi^{h}\right\|_{-1, \Gamma}^{2}\right) .
$$

Lemma I.6. Let $\mathcal{I}_{h}: \mathbf{C}(\Gamma) \rightarrow S^{h}$ denote the Lagrange interpolation operator then there holds

$$
\begin{aligned}
R_{4}^{h} \lesssim & h_{\max , \Gamma}\left\|\mathbf{u}-\mathbf{u}^{h}\right\|_{0, \Gamma}^{2}+h_{\max , \Gamma}\left\|\phi-\phi^{h}\right\|_{1 / 2, \Gamma}^{2} \\
& +\frac{h_{\max , \Gamma}}{h_{\min , \Gamma}}\left\|\mathcal{I}_{h} \phi-\phi\right\|_{1 / 2, \Gamma}^{2}+\frac{h_{\max , \Gamma}}{h_{\min , \Gamma}}\left\|\phi-\phi^{h}\right\|_{1 / 2, \Gamma}^{2},
\end{aligned}
$$

where $h_{\max , \Gamma}$ and $h_{\min , \Gamma}$ are the maximum and minimum length of the regular decomposition $\mathcal{S}_{\Gamma, \tilde{h}}$ of $\Gamma$, respectively.

Proof. Noting that $\frac{1}{\rho_{0} \omega^{2}} \frac{\partial p^{0}}{\partial \mathbf{n}}=\mathbf{u} \cdot \mathbf{n}-\frac{1}{\rho_{0} \omega^{2}}\left(\left(K^{\prime}-\frac{I}{2}\right)-\alpha W\right) \phi^{h}$ we obtain in each $s \in \mathcal{S}_{\Gamma, \tilde{h}}$

$$
\begin{aligned}
\|-\frac{1}{\rho_{0} \omega^{2}} & \frac{\partial p^{0}}{\partial n}+\mathbf{u}^{h} \cdot \mathbf{n}-\frac{1}{\rho_{0} \omega^{2}}\left(\left(K^{\prime}-\frac{I}{2}\right)-\alpha W\right) \phi^{h} \|_{0, s}^{2} \\
& \lesssim\left\|\left(\mathbf{u}-\mathbf{u}^{h}\right) \cdot \mathbf{n}\right\|_{0, s}^{2}+\left\|\left(K^{\prime}-\frac{I}{2}\right)\left(\phi-\phi^{h}\right)\right\|_{0, s}^{2}+\left\|\alpha W\left(\phi-\phi^{h}\right)\right\|_{0, s}^{2}
\end{aligned}
$$


Summing the estimate (32) over all elements $s \in \mathcal{S}_{\Gamma, \tilde{h}}$ we obtain

$$
R_{4}^{h} \lesssim h_{\max , \Gamma}\left(\left\|\rho_{0} \omega^{2}\left(\mathbf{u}-\mathbf{u}^{h}\right) \cdot \mathbf{n}\right\|_{0, \Gamma}^{2}+\left\|\left(K^{\prime}-\frac{I}{2}\right)\left(\phi-\phi^{h}\right)\right\|_{0, \Gamma}^{2}+\left\|\alpha W\left(\phi-\phi^{h}\right)\right\|_{0, \Gamma}^{2}\right),
$$

Since $K^{\prime}: H^{-1 / 2+\tilde{s}}(\Gamma) \rightarrow H^{-1 / 2+\tilde{s}}(\Gamma)$ and $W: H^{1 / 2+\tilde{s}}(\Gamma) \rightarrow H^{-1 / 2+\tilde{s}}(\Gamma)$ are continuous mappings for $\tilde{s} \in[-1 / 2,1 / 2]$, we have that

$$
\left\|\alpha W\left(\phi-\phi^{h}\right)\right\|_{0, \Gamma}^{2} \lesssim\left\|\phi-\phi^{h}\right\|_{1, \Gamma}^{2}, \quad\left\|\left(K^{\prime}-\frac{I}{2}\right)\left(\phi-\phi^{h}\right)\right\|_{0, \Gamma}^{2} \lesssim\left\|\phi-\phi^{h}\right\|_{0, \Gamma}^{2} .
$$

The triangle inequality gives

$$
\left\|\phi-\phi^{h}\right\|_{1, \Gamma}^{2} \leq\left\|\phi-\mathcal{I}_{h} \phi\right\|_{1, \Gamma}^{2}+\left\|\mathcal{I}_{h} \phi-\phi^{h}\right\|_{1, \Gamma}^{2},
$$

since $\left(\mathcal{I}_{h} \phi-\phi^{h}\right) \in \mathcal{B}^{h}$ we can apply the inverse Assumption I.4

$$
\left\|\mathcal{I}_{h} \phi-\phi^{h}\right\|_{1, \Gamma}^{2} \leq h_{\min , \Gamma}^{-1}\left\|\mathcal{I}_{h} \phi-\phi^{h}\right\|_{\frac{1}{2}, \Gamma}^{2} \leq h_{\min , \Gamma}^{-1}\left(\left\|\mathcal{I}_{h} \phi-\phi\right\|_{\frac{1}{2}, \Gamma}^{2}+\left\|\phi-\phi^{h}\right\|_{\frac{1}{2}, \Gamma}^{2}\right) .
$$

Thus

$$
R_{4}^{h} \lesssim h_{\max , \Gamma}\left(\left\|\mathbf{u}-\mathbf{u}^{h}\right\|_{0, \Gamma}^{2}+\left\|\phi-\phi^{h}\right\|_{0, \Gamma}^{2}\right)+\frac{h_{\max , \Gamma}}{h_{\min , \Gamma}}\left(\left\|\mathcal{I}_{h} \phi-\phi^{h}\right\|_{1 / 2, \Gamma}^{2}+\left\|\phi-\phi^{h}\right\|_{1 / 2, \Gamma}^{2}\right) .
$$

Now, we are ready to establish an upper bound for all indicators. Taking into account the results given in Lemmas I.3- I.6 it follows the following efficiency result of the error estimator for the formulation $\left(V P_{1}\right)$.

Theorem I.7. Let $(\mathbf{u}, \phi) \in \mathcal{H}_{1}$ be the solution of problem $\left(V P_{1}\right)$ and $\left(\mathbf{u}^{h}, \phi^{h}\right) \in \mathcal{H}_{1}^{h}$ be the solution of the discrete problem $\left(V P_{1}^{h}\right)$. Then there holds the following efficiency result

$$
\begin{aligned}
\left(R_{1}^{h}+R_{2}^{h}+R_{3}^{h}+R_{4}^{h}\right) \lesssim & \left\|\sigma\left(\mathbf{u}-\mathbf{u}^{h}\right)\right\|_{0, \Omega}^{2}+h_{\max , \Omega}\left\|\sigma\left(\mathbf{u}-\mathbf{u}^{h}\right)\right\|_{0, \Gamma}^{2} \\
& +h_{\max , \Omega}^{2}\left\|\mathbf{u}-\mathbf{u}^{h}\right\|_{0, \Omega}^{2}+h_{\max , \Omega}^{2}\|\mathbf{u}\|_{0, \Omega}^{2}+h_{\max , \Gamma}\left\|\left(\mathbf{u}-\mathbf{u}^{h}\right)\right\|_{0, \Gamma}^{2} \\
& +h_{\max , \Gamma}\left\|\phi-\phi^{h}\right\|_{0, \Gamma}^{2}+h_{\max , \Gamma}\left\|\phi-\phi^{h}\right\|_{-1, \Gamma}^{2} \\
& +\frac{h_{\max , \Gamma}}{h_{\min , \Gamma}}\left\|\mathcal{I}_{h} \phi-\phi\right\|_{1 / 2, \Gamma}^{2}+\frac{h_{\max , \Gamma}}{h_{\min , \Gamma}}\left\|\phi-\phi^{h}\right\|_{1 / 2, \Gamma}^{2},
\end{aligned}
$$

where $h_{\max , \Omega}$ is the maximum length of the regular decomposition $\mathcal{T}_{h}$ of $\Omega$ and $h_{\max , \Gamma}, h_{\min , \Gamma}$ are the maximum and minimum length of the regular decomposition $\mathcal{S}_{\Gamma, \tilde{h}}$ of $\Gamma$, respectively.

Remark I.8. Let us consider quasi-uniform meshes on $\Omega$ and their boundary $\Gamma$, i.e., meshes for which there exist constants $c_{1}, c_{2}>0$, independent of the meshsize, such that

$$
1 \leq \frac{h_{\max , \Omega}}{h_{\min , \Omega}} \leq c_{1}, \quad 1 \leq \frac{h_{\max , \Gamma}}{h_{\min , \Gamma}} \leq c_{2} .
$$

Using regularity assumptions on the solution, i.e., $(\mathbf{u}, \phi) \in\left[H^{2}(\Omega)\right]^{d} \times H^{3 / 2}(\Gamma)$ and the approximation properties of the Lagrangian interpolation operator yield

$$
\begin{aligned}
\left\|\phi-\mathcal{I}_{h} \phi\right\|_{1 / 2, \Gamma}^{2} & \lesssim h_{\max , \Gamma}^{2}\|\phi\|_{3 / 2, \Gamma}^{2}, \\
h_{\max , \Gamma}\left\|\phi-\phi^{h}\right\|_{0, \Gamma}^{2} & \lesssim h_{\max , \Gamma}^{4}\|\phi\|_{3 / 2, \Gamma}^{2},
\end{aligned}
$$

Copyright (c) 2000 John Wiley \& Sons, Ltd. Prepared using nmeauth.cls 


$$
h_{\max , \Gamma}\left\|\phi-\phi^{h}\right\|_{-1, \Gamma}^{2} \lesssim h_{\max , \Gamma}^{6}\|\phi\|_{3 / 2, \Gamma}^{2} .
$$

According to Carstensen [24, p. 318]) we expect that exists $h_{0}>0$ such that for all $h \leq h_{0}$

$$
c h_{\max , \Gamma}^{2} \leq\left\|\phi-\phi^{h}\right\|_{1 / 2, \Gamma}^{2}, \quad c h_{\max , \Omega}^{2} \leq\left\|\mathbf{u}-\mathbf{u}^{h}\right\|_{1, \Omega}^{2} .
$$

Thus, using (35) and (36)

$$
\begin{aligned}
\frac{h_{\max , \Gamma}}{h_{\min , \Gamma}}\left\|\mathcal{I}_{h} \phi-\phi\right\|_{1 / 2, \Gamma}^{2}+h_{\max , \Gamma}\left\|\phi-\phi^{h}\right\|_{0, \Gamma}^{2}+h_{\max , \Gamma}\left\|\phi-\phi^{h}\right\|_{-1, \Gamma}^{2} \\
\quad \lesssim h_{\max , \Gamma}^{2}\|\phi\|_{3 / 2, \Gamma}^{2}+h_{\max , \Gamma}^{4}\|\phi\|_{3 / 2, \Gamma}^{2}+h_{\max , \Gamma}^{6}\|\phi\|_{3 / 2, \Gamma}^{2} \\
\quad \lesssim h_{\max , \Gamma}^{2}\|\phi\|_{3 / 2, \Gamma}^{2} \\
\quad \lesssim\left\|\phi-\phi^{h}\right\|_{0, \Gamma}^{2},
\end{aligned}
$$

and

$$
\begin{aligned}
& \left\|\sigma\left(\mathbf{u}-\mathbf{u}^{h}\right)\right\|_{0, \Omega}^{2}+h_{\max , \Omega}\left\|\sigma\left(\mathbf{u}-\mathbf{u}^{h}\right)\right\|_{0, \Gamma}^{2}+h_{\max , \Omega}^{2}\left\|\mathbf{u}-\mathbf{u}^{h}\right\|_{0, \Omega}^{2} \\
& \quad+h_{\max , \Gamma}\left\|\left(\mathbf{u}-\mathbf{u}^{h}\right)\right\|_{0, \Gamma}^{2}+h_{\max , \Omega}^{2}\|\mathbf{u}\|_{0, \Omega}^{2} \\
& \lesssim\left\|\sigma\left(\mathbf{u}-\mathbf{u}^{h}\right)\right\|_{0, \Omega}^{2}+\left\|\mathbf{u}-\mathbf{u}^{h}\right\|_{1, \Omega}^{2} \lesssim\left\|\mathbf{u}-\mathbf{u}^{h}\right\|_{1, \Omega}^{2} .
\end{aligned}
$$

Together with Theorem I.7, (37) and (38) we get

$$
R_{1}^{h}+R_{2}^{h}+R_{3}^{h}+R_{4}^{h} \lesssim\left\|\mathbf{u}-\mathbf{u}^{h}\right\|_{1, \Omega}^{2}+\left\|\phi-\phi^{h}\right\|_{1 / 2, \Gamma}^{2} .
$$

For formulation $\left(V P_{2}\right)$ an analogous efficiency estimate can be shown and is omitted for brevity. 